\title{
ON THE STEINER 2-EDGE CONNECTED SUBGRAPH POLYTOPE
}

\author{
A. Rhida Mahjoub ${ }^{1}$ and Pierre Pesneau ${ }^{2}$
}

\begin{abstract}
In this paper, we study the Steiner 2-edge connected subgraph polytope. We introduce a large class of valid inequalities for this polytope called the generalized Steiner $F$-partition inequalities, that generalizes the so-called Steiner $F$-partition inequalities. We show that these inequalities together with the trivial and the Steiner cut inequalities completely describe the polytope on a class of graphs that generalizes the wheels. We also describe necessary conditions for these inequalities to be facet defining, and as a consequence, we obtain that the separation problem over the Steiner 2-edge connected subgraph polytope for that class of graphs can be solved in polynomial time. Moreover, we discuss that polytope in the graphs that decompose by 3 -edge cutsets. And we show that the generalized Steiner $F$-partition inequalities together with the trivial and the Steiner cut inequalities suffice to describe the polytope in a class of graphs that generalizes the class of Halin graphs when the terminals have a particular disposition. This generalizes a result of Barahona and Mahjoub [4] for Halin graphs. This also yields a polynomial time cutting plane algorithm for the Steiner 2-edge connected subgraph problem in that class of graphs.
\end{abstract}

Keywords. Polytope, Steiner 2-edge connected graph, Halin graph.

Mathematics Subject Classification. 05C85, 90C27.

\footnotetext{
Received December 1st, 2005. Accepted August 1st 2007.

1 LIMOS, CNRS, Univ. Blaise Pascal, Clermont Ferrand II, Complexe Sci. des Cézeaux, 63177 Aubière Cedex; present address: LAMSADE, CNRS, Univ. Paris-Dauphine, Place du Maréchal De Lattre de Tassigny, 75775 Paris Cedex 16, France mahjoub@lamsade.dauphine.fr

2 Université Bordeaux 1, IMB, 351, Cours de la Libération, 33405 Talence Cedex, France; pierre.pesneau@math.u-bordeaux1.fr 


\section{INTRODUCTION}

The design of cost-efficient survivable telecommunication networks is a major challenge with great economic impact. Survivable networks must satisfy certain connectivity requirements. A typical survivable condition is that between every pair of nodes of the network there are at least two edge-disjoint (node-disjoint) paths. In practice, there may exist distinguished nodes for which the survivable condition must be satisfied. In this paper we discuss this problem from a polyhedral point of view. The problem of designing general survivable telecommunication networks has been studied by Grötschel and Monma [18] and Grötschel et al. [1921]. Related works and applications can also be found in Bienstock et al. [5], Christofides and Whitlock [8], Erikson et al. [14], Monma et al. [30], Steiglitz et al. [32], Voss [33] and Winter [34,35].

A graph $G=(V, E)$ is said to be $k$-edge (resp. $k$-node) connected $(1 \leq k \leq$ $|V|-1$ ) if for every pair of nodes $i, j \in V$ there are at least $k$ edge-disjoint (resp. $k$ node-disjoint) paths from $i$ to $j$. Let $G=(V, E)$ be a graph and $\omega \in \mathbb{R}^{E}$ a weight vector associated with the edges of $G$. Given a subset of distinguished nodes $S \subseteq V$, called terminals, the Steiner 2-edge connected subgraph problem (STECSP) is the problem of finding a minimum weight subgraph of $G$ spanning $S$ such that between every two nodes $i, j \in S$, there are at least two edge-disjoint paths between $i$ and $j$.

Polyhedral combinatorics has been succesfully applied to obtain efficient cutting plane algorithms for combinatorial optimization problems. In this paper we discuss the polytope associated with the solutions to the STECSP. We introduce a large class of valid inequalities that generalizes the so-called Steiner $F$-partition inequalities. We show that these inequalities together with the trivial and the Steiner cut inequalities completely describe the polytope in a class of graphs that generalizes the wheels. We also describe necessary conditions for these inequalities to be facet defining, and as a consequence, we obtain that the separation problem over the Steiner 2-edge connected subgraph polytope for that class of graphs can be solved in polynomial time. Moreover, we discuss that polytope in the graphs that decompose by 3 -edge cutsets. And we show that generalized Steiner $F$-partition inequalities together with the trivial and the Steiner cut inequalities suffice to describe the polytope in a class of graphs that generalizes the class of Halin graphs when the terminals have a particular disposition. This generalizes a result of Barahona and Mahjoub [4] for Halin graphs. This also yields a polynomial time cutting plane algorithm for the Steiner 2-edge connected subgraph problem in that class of graphs.

The STECSP is NP-hard in general. Winter devised a linear time algorithm to solve the STECSP in series-parallel graphs [34] and in Halin graphs [35]. Grötschel and Monma [18] and Grötschel, Monma and Stoer [19-21] study the STECSP within the framework of a more general model. In particular, Grötschel and Monma [18] describe various classes of facets of the polytope associated with that model and Grötschel et al. [19-21] study further facets and devise cutting plane algorithms. A complete survey of that model can be found in $[22,25]$. 
Given a graph $G=(V, E)$ and a node subset $W \subseteq V$ of $G$, the set of edges having one endnode in $W$ and the other in $V \backslash W$ is called a cut and denoted by $\delta(W)$. If $W=\{v\}$ for some $v \in V$, then we write $\delta(v)$ for $\delta(W)$.

Let $G=(V, E)$ be a graph. Let $x(e)$ be a variable associated with each edge $e$. For an edge subset $F \subseteq E$, the $0-1$ vector $x^{F} \in \mathbb{R}^{E}$ with $x^{F}(e)=1$ if $e \in F$ and $x^{F}(e)=0$ if not, is called the incidence vector of $F$. For any subset of edges $T \subseteq E$, we define $x(T)=\sum_{e \in T} x(e)$.

The STECSP can be formulated as the following integer linear program.

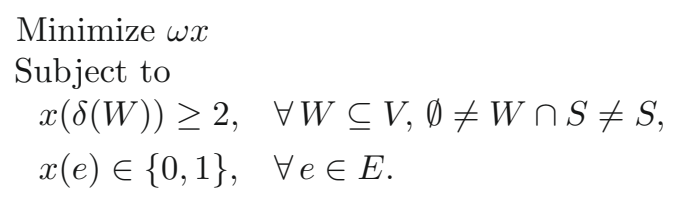

Inequalities (1.1) are called Steiner cut inequalities. The linear relaxation of the above formulation is obtained by replacing integrity constraints (1.2) by the trivial inequalities

$$
0 \leq x(e) \leq 1, \quad \forall e \in E
$$

Let

$$
\operatorname{STECSP}(G, S)=\operatorname{conv}\left\{x \in \mathbb{R}^{E} \mid x \text { satifies (1.1) and (1.2) }\right\}
$$

be the polytope associated with the $\operatorname{STECSP}$. The polytope $\operatorname{STECSP}(G, S)$ has been extensively investigated for $S=V$. In [28], Mahjoub gives a complete description of the $\operatorname{STECSP}(G, V)$ in series-parallel graphs. In [6], Boyd and Hao study a family of comb inequalities for the $\operatorname{STECSP}(G, V)$. In [4], Barahona and Mahjoub describe the $\operatorname{STECSP}(G, V)$ in Halin graphs. Fonlupt and Mahjoub $[15,16]$ study the linear relaxation $P(G)$ of the $\operatorname{STECSP}(G, V)$, that is the polytope given by inequalities (1.3) and (1.1). They introduce the notion of critical extreme points of $P(G)$. Roughly speaking, an extreme point of $P(G)$ is critical if it is fractional and the set of edges corresponding to its fractional values does not strictly contain the set of edges corresponding to the fractional values of another extreme point. They give a characterization of the critical extreme points, and, as a consequence, they obtain a characterization of the so-called perfectly 2-edge connected graphs, the graphs for which the polytope $P(G)$ is integer.

Baïou and Mahjoub [3] discuss the $\operatorname{STECSP}(G, S)$ and show that when the graph is series-parallel, $\operatorname{STECSP}(G, S)$ is given by the trivial and the Steiner cut inequalities. Didi Biha and Mahjoub [12] extend this to the Steiner $k$-edge connected subgraph polytope when $k$ is even (STECSP corresponds to the case where $k=2$ ). Recently, Kerivin and Mahjoub [27] extend this to the more general survivable network model when, with each node $v$ of the graph, it is associated a connectivity type $r(v)$. Actually, the problem, here, is to construct a minimum cost network such that between every pair of nodes $u, v$, there are at least $\min (r(u), r(v))$ edge-disjoint paths. Kerivin and Mahjoub [27] show that the trivial and the corresponding cut inequalities suffice to describe the polytope when the graph is series-parallel and the node types are all even. 
Related work can be found in [7,9-12,17]. In [9], Cornuéjols, Fonlupt and Naddef study the dominant of $\operatorname{STECSP}(G, S)$, and show that when $S=V$ and $G$ is series-parallel, the dominant is given by the nonnegativity inequalities and the cut inequalities. Fonlupt and Naddef [17] characterize the class of graphs for which the system given by these inequalities defines the convex hull of the incidence vectors of the tours of $G$ (a tour is a cycle going at least once through each node). In [7], Chopra considers the Steiner $k$-edge connected subgraph problem when multiple copies of an edge could be used. He gives a complete description of the associated polyhedron when $G$ is outerplanar and $k$ is odd. (A graph is outerplanar if it is planar and it can be embedded on the plane so that all nodes lie on the outermost face.) Didi Biha and Mahjoub [12] extend this to the more general class of series-parallel graphs. In [13] Didi Biha and Mahjoub study extensions of the concept of critical extreme points to the $k$-edge connected sybgraph polytope. Coullard et al. $[10,11]$ discuss the Steiner 2-node connected subgraph polytope. In [10], they describe the polytope for series-parallel graphs, and in [11] they describe the dominant of that polytope for the $W_{4}$-free graphs $\left(W_{4}\right.$ is the wheel on five nodes).

The paper is organized as follows. In the next section we introduce the class of generalized Steiner $F$-partition inequalities. In Section 3 we give a complete description of the polytope $\operatorname{STECSP}(G, S)$ on a class of graphs that generalizes the wheels and discuss facet conditions. In Section 4, we study the polytope $\operatorname{STECSP}(G, S)$ in the graphs that decompose by 3 -edge cutsets, and discuss some applications. In Section 5 we address the algorithmic aspect. And in Section 6 we give some concluding remarks.

The remainder of this section is devoted to more definitions and notations. If $G=(V, E)$ is a graph and $e$ is an edge between two nodes $u$ and $v$, then we write $e=u v$. If $W_{1}, \ldots, W_{q}$ are pairwise disjoint subsets of $V$, we let $\delta\left(W_{1}, \ldots, W_{q}\right)$ denote the set of edges between the sets $W_{1}, \ldots, W_{q}$. Note that if $W \subset V$, then $\delta(W)=\delta(W, V \backslash W)$. If $v_{1}, v_{2} \in V$, then we write $\delta\left(v_{1}, v_{2}\right)$ instead of $\delta\left(\left\{v_{1}\right\},\left\{v_{2}\right\}\right)$. If $W \subseteq V$, then we let $E(W)$ denote the set of edges having both endnodes in $W$. If $T \subseteq E$, then $V(T)$ will denote the set of the nodes of the edges of $T$.

Given an edge $e=u v \in E$, contracting $e$ consists of identifying $u$ and $v$ and of preserving all other vertices and of preserving all other adjacencies between vertices. Contracting a set of edges $T \subseteq E$ consists of contracting all the edges of $T$. We assume familiarity with basic definitions in polyhedral theory. Undefined polyhedral terminology and notation are consistent with that of Pulleyblank [31].

\section{Generalized Steiner F-partition inequalities}

In this section, we introduce a new class of valid inequalities for the STECSP $(G, V)$. As it will turn out, these inequalities generalize the so-called Steiner $F$ partition inequalities, and may have coefficients other than 0 and 1.

Let $G=(V, E)$ be a graph and $S \subseteq V$ a set of terminals. In [28] a family of valid inequalities for the $\operatorname{STECSP}(G, S)$ where $S=V$ has been introduced as 
follows. Consider a partition of $V$ into $V_{0}, V_{1}, \ldots, V_{p}$ and let $F \subseteq \delta\left(V_{0}\right)$ with $|F|$ odd. If we add the inequalities

$$
\begin{array}{ll}
x\left(\delta\left(V_{i}\right)\right) \geq 2 & 1 \leq i \leq p, \\
-x(e) \geq-1 & \forall e \in F, \\
x(e) \geq 0 & \forall e \in \delta\left(V_{0}\right) \backslash F,
\end{array}
$$

we obtain

$$
2 x(\Delta) \geq 2 p-|F|,
$$

where $\Delta=\delta\left(V_{0}, \ldots, V_{p}\right) \backslash F$. Dividing by 2 and rounding up the right-hand side, we obtain

$$
x(\Delta) \geq p-\left\lfloor\frac{|F|}{2}\right\rfloor .
$$

Inequalities of type (2.1) are called $F$-partition inequalities. Note that inequality (2.1) is also valid for $\operatorname{STECSP}(G, V)$ if $|F|$ is even. However, in this case, it is redundant. When $S \neq V$, if $V_{i} \cap S \neq \emptyset$ for $i=1, \ldots, p$, then it is straightforward to verify that $(2.1)$ is valid for $\operatorname{STECSP}(G, S)$. In that case, inequalities (2.1) are called Steiner F-partition inequalities.

In [2] Baïou, Barahona and Mahjoub show that the separation problem for $F$ partition inequalities can be solved in polynomial time when $F$ is fixed. Fonlupt and Mahjoub $[15,16]$ show that the so-called critical extreme points of the 2edge connected subgraph polytope can be separated in polynomial time, using $F$-partition inequalities. Moreover, $F$-partition inequalities have been shown to be very efficient in the framework of a cutting plane algorithm for solving both the 2-edge connected subgraph problem and the travelling salesman problem [26].

A Halin graph $G=(V, T \cup C)$ consists of a tree $T$ that has no degree-two node, together with a simple cycle $C$ whose nodes are the pendant nodes of $T$. The graph should be embeddeble in the plane with $C$ as the exterior face. These are examples of minimaly 3-connected graphs given by Halin [23]. Wheels are those Halin graphs with $T$ being a star. A wheel with $n+1$ nodes will be denoted by $W_{n}$.

Barahona and Mahjoub [4] prove that the trivial, cut and $F$-partition inequalities describe $\operatorname{STECSP}(G, S)$ when $G$ is a Halin graph and $S=V$. A natural question that arises is whether or not, the trivial, Steiner cut and Steiner $F$ partition inequalities suffice to completely describe the $\operatorname{STECSP}(G, S)$ when $G$ is a Halin graph and $S \neq V$. The answer to this question is, unfortunately, in the negative as shown by the following example.

Consider the wheel $W_{4}=(V, E)$ shown in Figure 1. Let $S=\left\{u_{1}, u_{2}, u_{3}\right\}$ and let $x \in \mathbb{R}^{E}$ be given by $x\left(e_{1}\right)=x\left(e_{2}\right)=x\left(e_{3}\right)=x\left(e_{4}\right)=x\left(f_{4}\right)=1 / 2$ and $x\left(f_{1}\right)=x\left(f_{2}\right)=x\left(f_{3}\right)=1$. It is not hard to see that $x$ satifies the trivial, Steiner cut and Steiner $F$-partition inequalities. Moreover, $x$ is an extreme point of the polytope described by these inequalities. This implies that further inequalities are needed to describe the polytope $\operatorname{STECSP}(G, S)$ on a wheel. In fact, it is easy to 


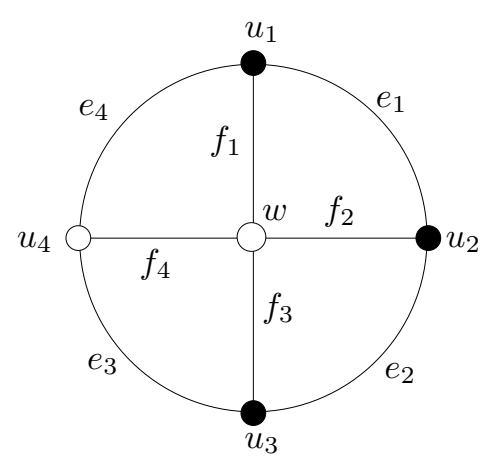

Figure 1.

see that $x$ does not satisfy the constraint

$$
2 x\left(e_{1}\right)+2 x\left(e_{2}\right)+x\left(e_{3}\right)+x\left(e_{4}\right)+x\left(f_{4}\right) \geq 4,
$$

that is valid for $\operatorname{STECSP}(G, S)$. In what follows, we show that this inequality is a special case of a more general class of valid inequalities for the $\operatorname{STECSP}(G, S)$.

\subsection{Generalized Steiner F-Partition inequalities}

Let $G=(V, E)$ be a graph and $S \subseteq V$ a set of terminals. Let $V_{0}, V_{1}, \ldots, V_{p}$ be a partition of $V$ such that for $i=1, \ldots, p$, if $V_{i} \cap S=\emptyset$ then $V_{i-1} \cap S \neq \emptyset$ and $V_{i+1} \cap S \neq \emptyset$ (the indices are taken modulo $p$ ). Let $F \subseteq \delta\left(V_{0}\right)$. Let $I \subset\{1, \ldots, p\}$ be the set of indices $i$ such that $V_{i} \cap S=\emptyset$. For $i \in I$, let

$$
E_{i}=\bigcup_{j \in I \cup\{0, i-1, i+1\}} \delta\left(V_{i}, V_{j}\right)
$$

Let

$$
\begin{aligned}
\Delta_{1} & =\bigcup_{i \in I} E_{i}, \\
\Delta_{2} & =\delta\left(V_{0}, V_{1}, \ldots, V_{p}\right) \backslash \Delta_{1} .
\end{aligned}
$$

Let $q=p-|I|$. Consider the following inequality.

$$
x\left(\Delta_{1} \backslash F\right)+2 x\left(\Delta_{2} \backslash F\right) \geq 2 q-\left\lfloor\frac{|F|}{2}\right\rfloor-\left\lfloor\frac{\left|F \cap \Delta_{2}\right|}{2}\right\rfloor .
$$

We have the following.

Theorem 2.1. Inequality (2.3) is valid for $\operatorname{STECSP}(G, S)$.

Proof. See [29].

In [29] it is shown that if $\left|F \cap \Delta_{2}\right|$ is even, then inequality (2.3) is redundant w.r.t. the trivial, Steiner cut and Steiner $F$-partition inequalities. Inequalities 


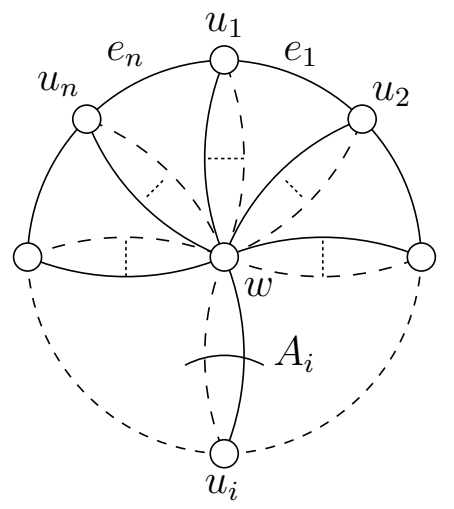

FiguRE 2.

of type (2.3) generalize the Steiner $F$-partition inequalities. Actually, the latter inequalities correspond to the case where $V_{i} \cap S \neq \emptyset$ for all $i \in 1, \ldots, p$.

Inequalities of type (2.3) will be called generalized Steiner F-partition inequalities. Note that these inequalities may have coefficients different from 0 and 1 . To the best of our knowledge, these are the first non-rank inequalities so far known for the $\operatorname{STECSP}(G, S)$, that is inequalities with coefficients different from 0 and 1.

Let $\boldsymbol{\Gamma}$ be the class of graphs $G=(V, E)$ such that $G$ is a wheel where the edges adjacent to the central node may be multiple edges. In the next section we shall show that the trivial and the Steiner cut inequalities together with the generalized Steiner $F$-partition inequalities completely describe the polytope $\operatorname{STECSP}(G, S)$ when $G$ is a graph of $\boldsymbol{\Gamma}$. To this end, let us first describe the generalized Steiner $F$-partition inequalities on $\boldsymbol{\Gamma}$ and give some notations specific to this class.

\subsection{Steiner $F$-Partition And Generalized Steiner $F$-Partition INEQUALITIES ON $\boldsymbol{\Gamma}$}

Given a graph $G=(V, E)$ of $\boldsymbol{\Gamma}$ on $n+1$ nodes, we let $V=\left\{w, u_{1}, \ldots, u_{n}\right\}$ where $w$ is the central node and $u_{1}, \ldots, u_{n}$ the nodes of the exterior cycle. We let $e_{i}$ denote the edge between $u_{i}$ and $u_{i+1}$ and $C=\left\{e_{1}, \ldots, e_{n}\right\}$. Let $A_{i}=\delta\left(u_{i}, w\right)$, for $i=1, \ldots, n$. If $i, j \in\{1, \ldots, n\}$, we denote by $C(i, j)$ the edge set $\left\{e_{i}, \ldots, e_{j-1}\right\}$ (where the indices are taken modulo $n$ ) (see Fig. 2). Note that if $\left|A_{i}\right|=1$ for $i=1, \ldots, n$, then $G$ is a wheel. We let $s=|S \cap V(C)|$, and denote by $u_{i_{1}}, \ldots, u_{i_{s}}$ the terminal nodes of $C$ such that $i_{1}<i_{2}<\cdots<i_{s}$.

Let $G=(V, E)$ be a graph of $\boldsymbol{\Gamma}$. Consider the generalized Steiner $F$-partition inequalities induced by partitions $V_{0}, \ldots, V_{p}$ of $V$ and $F \subset \delta\left(V_{0}\right)$, satisfying the following conditions:

$C_{1}: V_{0}=\{w\}$,

$C_{2}$ : the sets $V_{i}, i=1, \ldots, p$ are formed by consecutive nodes of the cycle $C$, $C_{3}$ : the order of the $V_{i}$ on the cycle $C$ corresponds to clockwise order, 


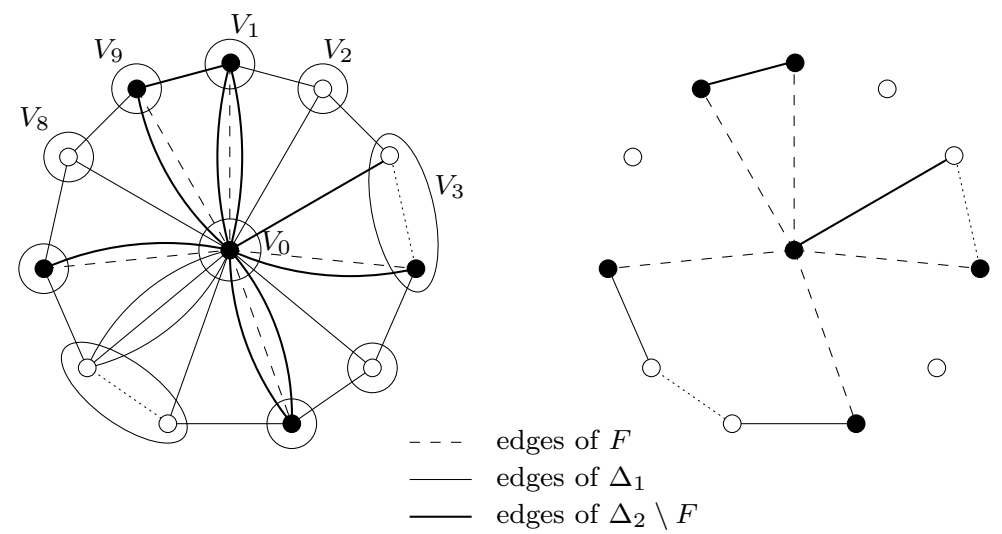

FiguRE 3.

$C_{4}: F \subset \bigcup_{V_{i} \cap S \neq \emptyset} \delta\left(V_{0}, V_{i}\right)$ and $\left|F \cap \delta\left(V_{0}, V_{i}\right)\right|=1$ for all $V_{i} \cap S \neq \emptyset$.

In consequence, we have

$$
\Delta_{1}=\bigcup_{i \in I} \delta\left(V_{i}\right), F \subset \Delta_{2} \text { and }|F|=q
$$

Since inequality (2.3) is redundant when $\left|\Delta_{2} \cap F\right|(=|F|)$ is even, we may suppose that $|F|$ is odd and hence $q$ is odd. Therefore inequality (2.3), in this case, can be rewritten as

$$
x\left(\Delta_{1}\right)+2 x\left(\Delta_{2} \backslash F\right) \geq q+1 .
$$

In order to illustrate inequality (2.4), consider the graph of $\boldsymbol{\Gamma}$ given on the left side of Figure 3. Here the terminal nodes correspond to the black nodes. We consider on that graph a partition $\left(V_{0}, V_{1}, \ldots, V_{9}\right)$. We have $q=5$. The edges of $F$ are indicated by dashed lines. The other edges are repesented by different types of lines in order to specify their coefficients in (2.4). The edges given in bold are those of $\Delta_{2} \backslash F$ with coefficient 2 . The edges given by solid lines are those of $\Delta_{1}$ with coefficient 1 , and the edges given by dashed and dotted lines are those with coefficient 0 , that is the edges that do not appear in the inequality. On the right side is shown a solution of the problem for which inequality (2.4) is tight.

(Also observe that inequality (2.2), corresponding to the graph of Fig. 1, is the inequality of type (2.4) induced by the partition $V_{0}, \ldots, V_{4}$ and the set $F$ where $V_{0}=\{w\}, V_{i}=\left\{u_{i}\right\}$ for $i=1, \ldots, 4$ and $\left.F=\left\{f_{1}, f_{2}, f_{3}\right\}.\right)$

Moreover, in case $w \in S$, consider the Steiner $F$-partition inequalities induced by partitions $V_{0}, \ldots, V_{p}$ of $V$ and edge set $F$ satisfying conditions:

$C_{1}^{\prime}: V_{1}=\{w\}$

$C_{2}^{\prime}$ : the sets $V_{i}, i \in\{0, \ldots, p\} \backslash\{1\}$ are formed by consecutive nodes of the cycle $C$,

$C_{3}^{\prime}:\left|V_{i} \cap S\right| \geq 1$ for $i=2, \ldots, p$,

$C_{4}^{\prime}: F \subset \delta\left(V_{0}\right)$ and $|F|=3$. 


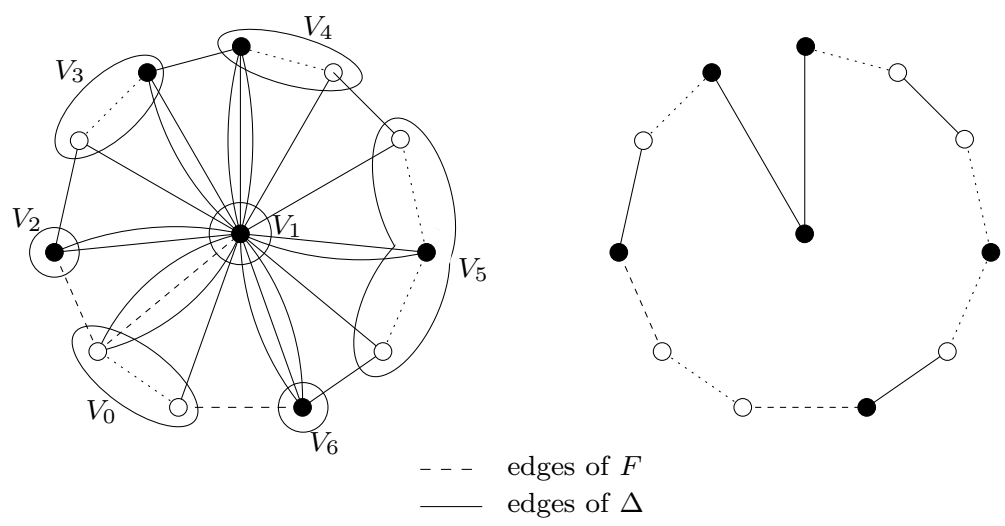

FIGURE 4.

Observe that $V_{i} \cap S \neq \emptyset$ for $i=1, \ldots, p$. By (2.1), these inequalities can be written as

$$
x(\Delta) \geq p-1 .
$$

Figure 4 illustrates inequality (2.1) for a graph of $\boldsymbol{\Gamma}$. On the left side of the figure, we consider a partition of the graph into seven sets, hence $p=6$. Here all the edges between the elements of the partition have coefficient 1 except those of $F$ (indicated by dashed lines) which have coefficient 0 . On the right side we show a solution for which inequality (2.1) is tight.

\section{The polytope $\operatorname{StECSP}(G, S)$ on $\Gamma$}

Throughout this section, given a graph $G=(V, E)$ of $\boldsymbol{\Gamma}$ and a node subset $S \subseteq V$ of terminals, we let

$$
T(G, S)=\{T \subseteq E \mid(V, T) \text { is a Steiner 2-edge connected subgraph of } G\} .
$$

Moreover, if $a x \geq \alpha$ is a facet defining inequality of $\operatorname{STECSP}(G, S)$, we let

$$
t_{a}=\left\{T \in T(G, S) \mid a x^{T}=\alpha\right\} .
$$

Our main result in this section is the following.

Theorem 3.1. Let $G=(V, E)$ be a graph of $\boldsymbol{\Gamma}$, and $S \subseteq V$ a set of terminals. Then $\operatorname{STECSP}(G, S)$ is defined by the trivial and Steiner cut inequalities together with inequalities (2.4) and (2.5).

The proof of this theorem will be given in Section 3.3. In what follows, we give a procedure that permits to construct a facet of $\operatorname{STECSP}(G, S)$ from a known one by the contraction of an edge. 


\subsection{Contraction operations}

Consider a graph $G=(V, E)$ and a node set $S$ of terminals. If $e=u v$ is an edge, we denote by $G_{e}=\left(V_{e}, E_{e}\right)$ the graph obtained from $G$ by contracting $e$. If $v^{\prime}$ is the new node that arises from the contraction, then we denote by $S_{e}$ the set of terminal nodes of $G_{e}$ such that $S_{e}=(S \backslash\{u, v\}) \cup v^{\prime}$ if $\{u, v\} \cap S \neq \emptyset$ and $S_{e}=S$ if not. Let $a x \geq \alpha$ be a facet defining inequality of $\operatorname{STECSP}(G, S)$ different from a trival inequality.

We have the following lemmas.

Lemma 3.1. Let $f=u v \in E$ be such that $a(f)=0$. Suppose that ax $\geq \alpha$ is valid for the polytope $\operatorname{STECSP}\left(G_{f}, S_{f}\right)$. Then ax $\geq \alpha$ defines a facet for $\operatorname{STECSP}\left(G_{f}, S_{f}\right)$.

Proof. W.l.o.g., we may suppose that $\operatorname{STECSP}(G, S)$ is full dimensional. Thus $\operatorname{STECSP}\left(G_{f}, S_{f}\right)$ so is. As $a x \geq \alpha$ is facet defining for $\operatorname{STECSP}(G, S)$, there are $|E|$ edge sets $T_{1}, \ldots, T_{|E|}$ that induce Steiner 2-edge connected subgraphs of $G$ such that $a x^{T_{i}}=\alpha$, for $i=1, \ldots,|E|$ and $x^{T_{1}}, \ldots, x^{T_{|E|}}$ are affinely independent. Let

$$
T_{i}^{\prime}= \begin{cases}T_{i} \backslash\{f\} & \text { if } f \in T_{i}, \\ T_{i} & \text { if not }\end{cases}
$$

for $i=1, \ldots,|E|$. Clearly, the sets $T_{i}^{\prime}, i=1, \ldots,|E|$, induce Steiner 2-edge connected subgraphs of $G_{f}$. Moreover we have $a x^{T_{i}^{\prime}}=\alpha$ for $i=1, \ldots,|E|$. Since $x^{T_{1}}, \ldots, x^{T_{|E|}}$ are affinely independent, there must exist $|E|-1$ sets among $T_{1}, \ldots, T_{|E|}$, whose incidence vectors are affinely independent. Since $a x \geq \alpha$ is valid for $\operatorname{STECSP}\left(G_{f}, S_{f}\right)$, it is then facet defining.

Lemma 3.2. Let $e_{i} \in C$ with $a\left(e_{i}\right)=0$. If $a^{\prime}$ is the restriction of a on $E_{e_{i}}$, then $a^{\prime} x \geq \alpha$ is valid for $\operatorname{STECSP}\left(G_{e_{i}}, S_{e_{i}}\right)$ if one of the following statements holds.

(1) $u_{i}, u_{i+1} \notin S$.

(2) $u_{i} \in S, u_{i+1} \notin S$ and for every edge $e$ of $A_{i+1}$, there is an edge $f$ of $A_{i}$ such that $a(e) \geq a(f)$.

(3) $u_{i}, u_{i+1} \in S$ and $\min \left\{a(e), e \in A_{i}\right\}=\min \left\{a(e), e \in A_{i+1}\right\}$.

Proof. We will show (1) and (2). The proof of (3) is similar.

Let $E^{*} \subseteq E_{e_{i}}$ such that $\left(V, E^{*}\right)$ is a Steiner 2-edge connected subgraph of $G_{e_{i}}$.

(1) Let $E_{1}^{*}=E^{*} \cup\left\{e_{i}\right\}$. Since $u_{i}, u_{i+1} \notin S, E_{1}^{*} \in T(G, S)$. Thus $a^{\prime} x^{E^{*}}=$ $a x^{E_{1}^{*}} \geq \alpha$, and hence $a^{\prime} x \geq \alpha$ is valid for $\operatorname{STECSP}\left(G_{e_{i}}, S_{e_{i}}\right)$.

(2) We distinguish three cases:

Case 1. $E^{*} \cap \delta\left(u_{i+1}\right)=\emptyset$. Since $u_{i+1} \notin S, E^{*} \in T(G, S)$, and thus $a^{\prime} x^{E^{*}}=$ $a x^{E^{*}} \geq \alpha$.

Case 2. $E^{*} \cap \delta\left(u_{i}\right) \neq \emptyset$ and $E^{*} \cap \delta\left(u_{i+1}\right) \neq \emptyset$. Thus $E_{2}^{*}=E^{*} \cup\left\{e_{i}\right\} \in T(G, S)$ and therefore $a^{\prime} x^{E^{*}}=a x^{E_{2}^{*}} \geq \alpha$.

Case 3. $E^{*} \cap \delta\left(u_{i}\right)=\emptyset$ and $E^{*} \cap \delta\left(u_{i+1}\right) \neq \emptyset$. Let $f_{2}^{*} \in E^{*} \cap A_{i+1}$, and $f_{1}^{*} \in A_{i}$ such that $a\left(f_{1}^{*}\right) \leq a\left(f_{2}^{*}\right)$, such an edge exists by hypothesis. Let $E_{3}^{*}=$ $\left(E^{*} \backslash\left\{f_{2}^{*}\right\}\right) \cup\left\{e_{i}, f_{1}^{*}\right\}$. It is easy to see that $E_{3}^{*} \in T(G, S)$. Moreover, as $a\left(e_{i}\right)=0$, we have that $a^{\prime} x^{E^{*}} \geq a x^{E_{3}^{*}} \geq \alpha$. 
In all cases, we have that $a^{\prime} x^{E^{*}} \geq \alpha$, which implies that $a^{\prime} x \geq \alpha$ is valid for $\operatorname{STECSP}\left(G_{e_{i}}, S_{e_{i}}\right)$.

\subsection{Structural Properties}

In this subsection, we shall describe some structural properties of the facet defining inequalities different from the trivial and the Steiner cut inequalities. These properties will be useful to show Theorem 3.1.

For this and the next subsections, we consider a graph $G=(V, E)$ of $\boldsymbol{\Gamma}$ on $n+1$ nodes. Thus $\operatorname{STECSP}(G, S)$ is full dimensional [29]. Suppose that $w \in S$ and $s \geq 2$. We also consider a facet defining inequality $a x \geq \alpha$ of $\operatorname{StECSP}(G, S)$ that is different from the trivial and the Steiner cut inequalities. Thus, if for an inequality $b x \geq \beta, b x^{T}=\beta$ for all $T \in t_{a}$, then $b x \geq \beta$ is a positive multiple of $a x \geq \alpha$

For $i=1, \ldots, n$, we let $f_{i}$ be a fixed edge of $A_{i}$ such that $a\left(f_{i}\right)=\min \{a(f) \mid f \in$ $\left.A_{i}\right\}$ for $i=1, \ldots, n$ and $E_{0}$ the set of edges $e$ such that $a(e)=0$.

We have the following lemmas given without proof. For the proof, see [29]. The first one is a direct consequence of the fact that $a x \geq \alpha$ is different from the trivial and Steiner cut inequalities.

\section{Lemma 3.3.}

(1) For every edge $e \in E$, there is an edge set $T \in t_{a}\left(T^{\prime} \in t_{a}\right)$ such that $e \in T$ $\left(e \notin T^{\prime}\right)$.

(2) For every node $v \in S$, there is an edge set $T \in t_{a}$ such that $|\delta(v) \cap T| \geq 3$.

(3) $a(e) \geq 0$ for all $e \in E$.

Lemma 3.4. Let $T \in t_{a}$ and $i \in\{1, \ldots, n\}$ such that $u_{i} \in S,\left|\delta\left(u_{i}\right) \cap T\right| \geq 3$ and for all $f \in A_{i}, a(f)>0$. Then the edges $e_{i}, e_{i-1}$ and $f_{i}$ may be supposed to be in $T$ if one of the following statements holds:

(1) $u_{i-1}, u_{i+1} \in S$.

(2) If $u_{i-1} \notin S$ (resp. $u_{i+1} \notin S$ ) then either $a\left(e_{i-1}\right)>0$ (resp. $\left.a\left(e_{i}\right)>0\right)$, or $a\left(e_{i-1}\right)=0$ and $a\left(f_{i-1}\right)=0$ (resp. $a\left(e_{i}\right)=0$ and $\left.a\left(f_{i+1}\right)=0\right)$.

Lemma 3.5. Let $T \in t_{a}$ and $i \in\{1, \ldots, n\}$ such that $u_{i} \in S$ and $e_{i}, e_{i+1}, f \in T$ for some $f \in A_{i}$ where $a(f)>0$. Then $C \subset T$, if one of the following statements holds:

(1) $u_{i-1}, u_{i+1} \in S$.

(2) $a\left(e_{i-1}\right)>0$ if $u_{i-1} \notin S$ (resp. $a\left(e_{i}\right)>0$ if $\left.u_{i+1} \notin S\right)$.

Lemma 3.6. Let $j \in\{1, \ldots, s\}$ such that $\left|A_{i_{j}} \cap E_{0}\right|=1$. Let $T \in t_{a}$ such that $f_{i_{j}} \notin T$. Then $A_{i_{j}} \cap T=\emptyset$. Moreover, if $a\left(e_{i_{j}-1}\right)>0$ (resp. $a\left(e_{i_{j}}\right)>0$ ) then $C\left(i_{j-1}, i_{j}\right) \subset T$ (resp. $\left.C\left(i_{j}, i_{j+1}\right) \subset T\right)$.

Lemma 3.7. Suppose that $C \cap E_{0}=\emptyset$. Then $\left|A_{i_{j}} \cap E_{0}\right|=1$ for all $j \in\{1, \ldots, s\}$.

Lemma 3.8. Suppose that $C \cap E_{0}=\emptyset$ and $s \geq 3$. Then there is $\beta>0$ such that

(1) $a\left(C\left(i_{j}, i_{j+1}\right)\right)=\beta$ for every $j \in\{1, \ldots, s\}$,

(2) if $j \in\{1, \ldots, s\}$ and $\left|A_{i_{j}}\right| \geq 2$, then $a(f)=\beta$ for all $f \in A_{i_{j}} \backslash\left\{f_{i_{j}}\right\}$. 
Lemma 3.9. Suppose $C \cap E_{0}=\emptyset$ and $s \geq 3$. Let $i \in\{1, \ldots, n\}$ such that $u_{i} \notin S$. Then

(1) $a(f)=\beta / 2$ for all $f \in A_{i}$,

(2) $u_{i+1} \in S$,

(3) $a\left(e_{i-1}\right)=a\left(e_{i}\right)=\beta / 2$,

where $\beta$ is the scalar introduced in Lemma 3.8.

\subsection{Proof of Theorem 3.1}

The proof is by induction on the number of nodes. The theorem is true for a graph of $\boldsymbol{\Gamma}$ on three nodes. In fact, in this case, the graph is series-parallel and, as shown by Baïou and Mahjoub [3], the $\operatorname{STECSP}(G, S)$ is then completely described by the trivial and the Steiner cut inequalities. Suppose the theorem is true for any graph of $\boldsymbol{\Gamma}$ with no more than $n$ nodes and suppose $G$ has exactly $n+1$ nodes.

Let $a x \geq \alpha$ be a facet defining inequality of $\operatorname{STECSP}(G, S)$ different from the trivial and the Steiner cut inequalities. We will show that $a x \geq \alpha$ is necessarily of type either (2.4) or (2.5). To this end, let us first note that, if there is an edge $f \in E$ with $a(f)=0$ that satisfies the conditions of Lemma 3.1, then $a x \geq \alpha$ defines a facet of the polytope associated with the graph obtained by contracting $f$. By the induction hypothesis, it follows that $a x \geq \alpha$ is of type (2.4). Thus, for the remainder of the proof, we suppose that no edge $e$ of $E$ with $a(e)=0$ satisfies the conditions of Lemma 3.1. As a consequence, by Lemma 3.2 we have the following.

\section{Claim 3.1.}

(1) If $u_{i}, u_{i+1} \notin S$ for $i \in\{1, \ldots, n\}$, then $a\left(e_{i}\right)>0$.

(2) If $u_{i} \in S, u_{i+1} \notin S$ and $a\left(e_{i}\right)=0$ for $i \in\{1, \ldots, n\}$, then $a\left(f_{i+1}\right)<a\left(f_{i}\right)$.

(3) If $u_{i}, u_{i+1} \in S$ for $i \in\{1, \ldots, n\}$, then $a\left(f_{i}\right) \neq a\left(f_{i+1}\right)$.

We shall suppose $w \in S$, the proof when $w \notin S$ is similar. If either $s=0$ or $s=1$, then it is not hard to see that, in this case, $a(e)=0$ for all $e \in E$, which is impossible. In the rest of the proof we suppose $s \geq 2$.

We distinguish two cases.

Case 1. $a(e)>0$ for all $e \in C$.

Suppose first that $s=2$ and hence $V(C) \cap S=\left\{u_{i_{1}}, u_{i_{2}}\right\}$. As $C \cap E_{0}=\emptyset$, by Lemma 3.7 we have $A_{i_{1}} \cap E_{0}=\left\{f_{i_{1}}\right\}$ and $A_{i_{2}} \cap E_{0}=\left\{f_{i_{2}}\right\}$. Now by Lemma 3.3 (1), there must exist a set $T \in t_{a}$ such that $f_{i_{1}} \notin T$. From Lemma 3.6, it follows that $C \subseteq T$. Let $T_{1}=\left(T \backslash C\left(i_{1}, i_{2}\right)\right) \cup\left\{f_{i_{1}}, f_{i_{2}}\right\}$. Obviously, $T_{1} \in T(G, S)$. This implies that $a(e)=0$ for all $e \in C\left(i_{1}, i_{2}\right)$, a contradiction. In consequence, if $|V(C) \cap S|=2$, then $\operatorname{STECSP}(G, S)$ is given by the trivial and the Steiner cut constraints.

Now suppose that $|V(C) \cap S| \geq 3$. Since $C \cap E_{0}=\emptyset$, by Lemma 3.7, it follows that $a\left(f_{i_{j}}\right)=0$ for $j=1, \ldots, s$. Moreover, as $s \geq 3$, from Lemma 3.8 (2) there is $\beta>0$ such that $a(f)=\beta$ for all $f \in A_{i_{j}} \backslash\left\{f_{i_{j}}\right\}$ and $j=1, \ldots, s$. In addition, by Lemma $3.9(2)$ if a node $u_{i}$ is not a terminal, then $u_{i-1}$ and $u_{i+1}$ are 
terminals. Hence by Lemma $3.9(3) a(u v)=\beta / 2$ for every edge $u v \in C$ such that $|\{u, v\} \cap S|=1$. Also by Lemma $3.9(1), a(e)=\beta / 2$ for all $e \in A_{i}$. Finally, if $u v$ is an edge of $C$ such that $u$ and $v$ are terminals, then by Lemma 3.8 (1), we have that $a(u v)=\beta$. Altogether, we then obtain

$$
a(e)= \begin{cases}\beta & \text { if } e=u v \in C \text { and } u, v \in S \\ \beta / 2 & \text { if } e=u v \in C \text { and }|\{u, v\} \cap S|=1, \\ \beta / 2 & \text { if } e \in A_{i} \text { and } u_{i} \notin S \\ \beta & \text { if } e \in A_{i_{j}} \backslash\left\{f_{i_{j}}\right\} \text { and } j \in\{1, \ldots, s\} \\ 0 & \text { if } e \in\left\{f_{i_{1}}, \ldots, f_{i_{s}}\right\}\end{cases}
$$

This implies that $a x \geq \alpha$ is an inequality of type (2.4) where the elements of the partition are given by the nodes of the graph and $F=\left\{f_{i_{1}}, \ldots, f_{i_{s}}\right\}$.

Case 2. $a\left(e_{i}\right)=0$ for some $i \in\{1, \ldots, n\}$.

By Claim 3.1 (1) it follows that $\left\{u_{i}, u_{i+1}\right\} \cap S \neq \emptyset$. In what follows, we consider the case where $\left|\left\{u_{i}, u_{i+1}\right\} \cap S\right|=1$. The case where $u_{i}, u_{i+1} \in S$ can be treated along the same line.

Suppose that $u_{i} \in S, u_{i+1} \notin S$. (The case $u_{i} \notin S, u_{i+1} \in S$ is similar.) We will suppose $u_{i}=u_{i_{1}}$. Also we let $T^{*}=\left(C \backslash\left\{e_{i_{1}}\right\}\right) \cup\left\{f_{i_{1}}, f_{i_{1}+1}\right\}$.

\section{Claim 3.2.}

(1) $a(f)>0$ for all $f \in A_{i_{1}}$.

(2) $T^{*} \in t_{a}$.

Proof. (1) If there is an edge $f \in A_{i_{1}}$ with $a(f)=0$, then $a(g) \geq a(f)$ for all $g \in A_{i_{1}+1}$, which contradicts Claim 3.1 (2).

(2) By Lemma $3.3(1)$, there is a set $T \in t_{a}$ such that $e_{i_{1}} \notin T$. As $u_{i_{1}} \in S$ and consequently, $\left|T \cap \delta\left(u_{i_{1}}\right)\right| \geq 2$, we may suppose that $f_{i_{1}} \in T$. If $A_{i_{1}+1} \cap T=\emptyset$, then $\left(T \backslash\left\{f_{i_{1}}\right\}\right) \cup\left\{e_{i_{1}}, f\right\} \in T(G, S)$ and therefore $a(f) \geq a\left(f_{i_{1}}\right)$ for all $f \in A_{i_{1}+1}$. But this contradicts again Claim 3.1 (2). In consequence, $A_{i_{1}+1} \cap T \neq \emptyset$. And thus we may suppose that $f_{i_{1}+1} \in T$. Now we claim that $C \backslash\left\{e_{i_{1}}\right\} \subset T$. Indeed, if this is not the case, then there must exist $k \in\{1, \ldots, n\} \backslash\left\{i_{1}+1\right\}$ such that $e_{k-1} \notin T$ and $C\left(k, i_{1}\right) \subset T$. Using the same arguments as above, we can show that $A_{k} \cap T \neq \emptyset$. (Note that $k$ may be equal to $i_{1}$, however, in this case we should have $\left.\left|A_{i_{1}} \cap T\right| \geq 2\right)$. Thus $\left(T \backslash\left\{f_{i_{1}}\right\}\right) \cup\left\{e_{i_{1}}\right\} \in T(G, S)$ and hence $a\left(f_{i_{1}}\right)=0$, contradicting (1). Thus $C \backslash\left\{e_{i_{1}}\right\} \subset T$ and hence $T^{*} \in t_{a}$.

\section{Claim 3.3.}

(1) $a(f)>0$ for all edge $f$ in $\delta(w)$ different from $f_{i_{1}+1}$.

(2) $a\left(f_{i_{1}+1}\right)=0$.

Proof. (1) Suppose $a(\bar{f})=0$ for some $\bar{f} \in \delta(w) \backslash\left\{f_{i_{1}+1}\right\}$. From Claim $3.2(2)$, it follows that $\left(T^{*} \backslash\left\{f_{i_{1}}\right\}\right) \cup\left\{e_{i_{1}}, f_{i_{1}+1}\right\}$ belongs to $T(G, S)$. Hence $a\left(f_{i_{1}}\right)=0$, contradicting Claim $3.2(1)$.

(2) As $w \in S$, by Lemma 3.3 (2) there must exist a set $T \in t_{a}$ such that $|\delta(w) \cap T| \geq 3$. 
We first show that $A_{i_{1}+1} \cap T \neq \emptyset$. Suppose, on the contrary, that $A_{i_{1}+1} \cap T=\emptyset$. If $|T \cap \delta(w)|=3$, then it is easy to see that there is an edge $f \in \delta(w) \cap T$ $\left(f \notin A_{i_{1}+1}\right)$ such that $T \backslash\{f\} \in T(G, S)$. But this implies that $a(f)=0$, which contradicts (1). Thus $|\delta(w) \cap T| \geq 4$. Let $k_{1}, k_{2}, k_{3} \in\{1, \ldots, n\} \backslash\left\{i_{1}+1\right\}$ such that $k_{1} \leq k_{2} \leq k_{3}, A_{k_{j}} \cap T \neq \emptyset$, for $j=1, \ldots, 3$ and $\left|T \cap\left(A_{k_{1}} \cup A_{k_{2}} \cup A_{k_{3}}\right)\right| \geq 3$. If $k_{1}=k_{2}=k_{3}$, then the set $T \backslash\left\{f_{k_{1}}\right\}$ still induces a feasible solution for the STECSP. But this implies that $a\left(f_{k_{1}}\right)=0$, contradicting (1). Suppose now that $k_{1}<k_{2}$ and hence $f_{k_{1}}, f_{k_{2}} \in T$. The case where $k_{1}=k_{2}$ and $k_{2}<k_{3}$ can be treated along the same line. We may also suppose, w.l.o.g., that $A_{i} \cap T=\emptyset$ for $i \in\left\{k_{1}+1, \ldots, k_{3}-1\right\} \backslash\left\{k_{2}\right\}$. If $C\left(k_{1}, k_{3}\right) \subset T$, then $T \backslash\left\{f_{k_{2}}\right\} \in T(G, S)$ and hence $a\left(f_{k_{2}}\right)=0$, contradicting (1). Thus we may suppose that $C\left(k_{1}, k_{2}\right) \not \subset T$. The case when $C\left(k_{2}, k_{3}\right) \not \subset T$ is similar. Therefore, $u_{i} \notin S$ for all $i \in\left\{k_{1}+1, \ldots, k_{2}-1\right\}$. Let $T^{\prime}=\left(T \backslash\left\{f_{k_{1}}, f_{k_{2}}\right\}\right) \cup C\left(k_{1}, k_{2}\right)$. As $|\delta(w) \cap T| \geq 4$, we have $T^{\prime} \in T(G, S)$. In fact, it is clear that all Steiner cut inequalities different from the one induced by $\{w\}$ are satisfied by $x^{T^{\prime}}$. Now, since $|\delta(w) \cap T| \geq 4$, the Steiner cut inequality induced by $\{w\}$ is also satisfied. In consequence, we get $a\left(f_{k_{1}}\right)+a\left(f_{k_{2}}\right) \leq a\left(C\left(k_{1}, k_{2}\right)\right)$.

On the other hand, from Claim $3.2(2)$ we have that $\left(T^{*} \backslash C\left(k_{1}, k_{2}\right)\right) \cup\left\{f_{k_{1}}, f_{k_{2}}\right\}$ belongs to $T(G, S)$. Thus $a\left(C\left(k_{1}, k_{2}\right)\right) \leq a\left(f_{k_{1}}\right)+a\left(f_{k_{2}}\right)$, and hence

$$
a\left(C\left(k_{1}, k_{2}\right)\right)=a\left(f_{k_{1}}\right)+a\left(f_{k_{2}}\right) .
$$

Furthermore note that $\left(T^{*} \backslash\left(C\left(k_{1}, k_{2}\right) \cup\left\{f_{i_{1}}, f_{i_{1}+1}\right\}\right)\right) \cup\left\{e_{i_{1}}, f_{k_{1}}, f_{k_{2}}\right\}$ belongs to $T(G, S)$. Since $a\left(e_{i_{1}}\right)=0$, by $(3.1)$, it follows that $a\left(f_{i_{1}}\right)=0$, a contradiction.

Thus $A_{i_{1}+1} \cap T \neq \emptyset$ and, in consequence, we may suppose that $f_{i_{1}+1} \in T$. Moreover, since $a\left(e_{i_{1}}\right)=0$, we may also suppose that $e_{i_{1}} \in T$. If there is a further edge of $A_{i_{1}+1}$, say $\bar{f}$, that belongs to $T$, then as $u_{i_{1}} \in S$, we have $T \backslash\{\bar{f}\} \in T(G, S)$. Hence $a(\bar{f})=0$, which is again impossible by (1). Thus $A_{i_{1}+1} \cap T=\left\{f_{i_{1}+1}\right\}$. Let $k \in\{1, \ldots, n\} \backslash\left\{i_{1}+1\right\}$ such that $A_{k} \cap T \neq \emptyset$ and $A_{l} \cap T=\emptyset$ for all $l \in\left\{i_{1}+2, \ldots, k-1\right\}$. Note that, as $|\delta(w) \cap T| \geq 3, k$ exists. Also note that $k$ cannot coincide with $i_{1}$. Indeed, if $k=i_{1}$, as $|T \cap \delta(w)| \geq 3$, there are at least two edges, say $g_{1}, g_{2}$ in $A_{i_{1}} \cap T$. But, this implies that $T \backslash\left\{g_{i}\right\} \in T(G, S)$, for $i=1,2$, and hence $a\left(g_{1}\right)=a\left(g_{2}\right)=0$, contradicting (1). Moreover, since $|T \cap \delta(w)| \geq 3$ and $u_{i_{1}} \in S$, there must exist $k^{\prime} \in\left\{k, \ldots, i_{1}\right\}$ such that $A_{k^{\prime}} \cap T \neq \emptyset$ and $C\left(k^{\prime}, i_{1}\right) \subset T$. So, we may suppose that $f_{k^{\prime}} \in T$. Note that $k^{\prime}$ can be equal to $i_{1}$. We have $k \neq k^{\prime}$. In fact, if $k=k^{\prime}$, then $T \backslash\left\{f_{k}\right\} \in T(G, S)$ and hence $a\left(f_{k}\right)=0$, which contradicts (1).

Now, we will show that $a\left(f_{i_{1}+1}\right)=0$. Consider first the case where $C\left(i_{1}+1, k\right) \subset$ $T$. Then clearly, $T \backslash\left\{f_{i_{1}+1}\right\} \in T(G, S)$ and hence $a\left(f_{i_{1}+1}\right)=0$. Suppose now that $C\left(i_{1}+1, k\right) \not \subset T$. As $A_{l} \cap T=\emptyset$ for all $l \in\left\{i_{1}+2, \ldots, k-1\right\}$, we have that $u_{l} \notin S$ for all $l \in\left\{i_{1}+1, \ldots, k-1\right\}$. Moreover, we have that $C\left(k, k^{\prime}\right) \not \subset T$. For otherwise, $T \backslash\left\{f_{k^{\prime}}\right\}$ would be in $T(G, S)$ and hence $a\left(f_{k^{\prime}}\right)=0$, contradicting (1). As by $(1) a\left(f_{k}\right)>0$, there must exist $k^{\prime \prime} \in\left\{k, \ldots, k^{\prime}-1\right\}$ and an edge $g_{k^{\prime \prime}}$ such that $g_{k^{\prime \prime}} \in A_{k^{\prime \prime}} \cap T$ and $C\left(k, k^{\prime \prime}\right) \subset T$. If not, then the set $T \backslash\left\{f_{k}\right\}$ would be in $T(G, S)$, and therefore $a\left(f_{k}\right)=0$, a contradiction. Now, it is not hard to see that 
the set $\left(T \backslash\left\{f_{i_{1}+1}, f_{k}\right\}\right) \cup C\left(i_{1}+1, k\right)$ belongs to $T(G, S)$, and in consequence

$$
a\left(f_{i_{1}+1}\right)+a\left(f_{k}\right) \leq a\left(C\left(i_{1}+1, k\right)\right)
$$

Since $u_{l} \notin S$ for all $l \in\left\{i_{1}+1, \ldots, k-1\right\},\left(T^{*} \backslash\left(C\left(i_{1}+1, k\right) \cup\left\{f_{i_{1}+1}\right\}\right)\right) \cup\left\{f_{k}\right\}$ induces a solution of STECSP. This yields

$$
a\left(f_{i_{1}+1}\right)+a\left(C\left(i_{1}+1, k\right)\right) \leq a\left(f_{k}\right)
$$

By (3.2) and (3.3), it follows that $a\left(f_{i_{1}+1}\right)=0$ which completes the proof of our claim.

\section{Claim 3.4.}

(1) If $u_{j} \in S \backslash\left\{u_{i_{1}}\right\}$, then $a\left(e_{j}\right)>0$.

(2) If $u_{j} \in S \backslash\left\{u_{i_{2}}\right\}$, then $a\left(e_{j-1}\right)>0$.

(3) $i_{2}=i_{1}+2$ and $a\left(e_{i_{2}-1}\right)=0$.

Proof. We only show (1) and (3). The proof for (2) is similar to that of (1).

(1) Suppose $a\left(e_{j}\right)=0$. If $u_{j+1} \notin S$, then by Claim $3.3(2)$, we have that $a\left(f_{j+1}\right)=0$, contradicting Claim $3.3(1)$.

So suppose that $u_{j+1} \in S$. By Lemma $3.3(1)$, there is a set $\bar{T} \in t_{a}$ that does not contain $e_{j}$. Thus we can suppose, w.l.o.g., that $f_{j}$ and $f_{j+1}$ belong to $\bar{T}$. If $C \backslash\left\{e_{j}\right\} \not \subset \bar{T}$, then we have $\left|\left(\delta(w) \backslash\left\{f_{i_{j}}, f_{i_{j}+1}\right\}\right) \cap \bar{T}\right| \geq 2$ and, in consequence, $\left(\bar{T} \backslash\left\{f_{i_{j}}, f_{i_{j}+1}\right\}\right) \cup\left\{e_{j}\right\} \in T(G, S)$. But this implies $a\left(f_{i_{j}}\right)=a\left(f_{i_{j}+1}\right)=0$, contradicting Claim 3.3 (1). Thus $C \backslash\left\{e_{j}\right\} \subset \bar{T}$, and therefore $\left(\bar{T} \backslash\left\{f_{i_{j}}\right\}\right) \cup$ $\left\{e_{j}, f_{i_{1}+1}\right\} \in T(G, S)$. Hence $a\left(f_{i_{j}}\right) \leq a\left(e_{j}\right)+a\left(f_{i_{1}+1}\right)=0$, which contradicts again Claim 3.3 (1).

(3) Suppose, on the contrary, that $i_{2}=i_{1}+l$ with $l \geq 3$. Then $a\left(e_{i}\right)>0$ for $i=i_{1}+1, \ldots, i_{1}+l-1$. In fact, this is clear for $e_{i_{1}+1}, \ldots, e_{i_{1}+l-2}$ by Claim 3.1 (1). Now, if $a\left(e_{i_{1}+l-1}\right)=0$, then by Claim $3.3(2)$, it follows that $a\left(f_{i_{1}+l-1}\right)=0$, contradicting Claim 3.3 (1).

As $u_{i_{2}} \in S$, by Lemma $3.3(2)$ there is an edge set $\tilde{T} \in t_{a}$ such that $\left|\delta\left(u_{i_{2}}\right) \cap \tilde{T}\right| \geq$ 3. As by (1) $a\left(e_{i_{2}}\right)>0$, from Lemmas 3.4 and 3.5, it follows that $C \cup\left\{f_{i_{2}}\right\} \subset \tilde{T}$. Now, since $u_{i_{1}+1}, \ldots, u_{i_{2}-1} \notin S$, we have that $\left(\tilde{T} \backslash C\left(i_{1}+1, i_{2}\right)\right) \cup\left\{f_{i_{1}+1}\right\} \in$ $T(G, S)$. As by Claim $3.3(2) a\left(f_{i_{1}+1}\right)=0$, this implies that $a\left(e_{i_{1}+1}\right)=\cdots=$ $a\left(e_{i_{2}-1}\right)=0$, a contradiction and thus $i_{2}=i_{1}+2$.

Now, we are going to show that $a\left(e_{i_{2}-1}\right)=0$. On the contrary, if $a\left(e_{i_{2}-1}\right)>0$, by using the same arguments as above, we obtain that $C \cup\left\{f_{i_{2}}\right\} \subset \tilde{T}$. Since $\left(\tilde{T} \backslash\left\{e_{i_{2}-1}\right\}\right) \cup\left\{f_{i_{1}+1}\right\} \in T(G, S)$, we get $a\left(e_{i_{2}-1}\right)=0$, a contradiction.

Claim 3.5. There is exactly one node $u_{i}$ not in $S$, namely $u_{i_{1}+1}$.

Proof. Assume the contrary. Since $i_{2}=i_{1}+2$, there must exist $l \in\{2, \ldots, s\}$ such that $V\left(C\left(i_{2}, i_{l}\right)\right) \subset S$ and $u_{i_{l}+1} \notin S$. Note that $l$ may be equal to 2 . As $u_{i_{l}} \in S$, by Lemma $3.3(2)$, there is an edge set $T_{1} \in t_{a}$ such that $\left|T_{1} \cap \delta\left(u_{i_{l}}\right)\right| \geq$ 3. As by Claim 3.4 (1) $a\left(e_{i_{l}}\right)>0$, it follows from Lemmas 3.4 and 3.5, that $C \cup\left\{f_{i_{l}}\right\} \subset T_{1}$. Now, by considering $u_{i_{l+1}}$ instead of $u_{i_{l}}$, we can show similarly 
that $a\left(e_{i_{l+1}-1}\right)>0$ and there is a set $T_{2} \in t_{a}$ such that $C \cup\left\{f_{i_{l+1}}\right\} \subset T_{2}$. As by Claim $3.3(2), a\left(f_{i_{1}+1}\right)=0$, we may suppose that $f_{i_{1}+1} \in T_{1} \cap T_{2}$. Consequently, we have $T_{1}=C \cup\left\{f_{i_{1}+1}, f_{i_{l}}\right\}$ and $T_{2}=C \cup\left\{f_{i_{1}+1}, f_{i_{l+1}}\right\}$. Since by Claim 3.2 (2), $T^{*}=\left(C \backslash\left\{e_{i_{1}}\right\}\right) \cup\left\{f_{i_{1}}, f_{i_{1}+1}\right\}$ belongs to $t_{a}$ and $a\left(e_{i_{1}}\right)=0$, it follows that $a\left(f_{i_{l}}\right)=a\left(f_{i_{l+1}}\right)=a\left(f_{i_{1}}\right)$. Moreover as $\left(T_{1} \backslash\left\{f_{i_{l}}\right\}\right) \cup\{f\} \in T(G, S)$ for all $f \in A_{k}$, $k \in\left\{i_{l}+1, \ldots, i_{l+1}-1\right\}$, we get

$$
a(f) \geq a\left(f_{i_{l}}\right) \text { for all } f \in A_{k}, k=i_{l}+1, \ldots, i_{l+1}-1 .
$$

On the other hand, there must exist an edge set $T_{3} \in t_{a}$ such that $\left|T_{3} \cap\left\{e_{i_{l}}, e_{i_{l+1}-1}\right\}\right|=1$. For otherwise, for every set $T$ of $t_{a}, x^{T}$ would verify the equation $x\left(e_{i_{l}}\right)-x\left(e_{i_{l+1}-1}\right)=0$. However, since $a \geq 0$, this equation cannot be a positive multiple of $a x=\alpha$, a contradiction.

W.l.o.g., we may suppose that $e_{i_{l}} \in T_{3}$ and $e_{i_{l+1}-1} \notin T_{3}$. Hence $A_{i_{l+1}} \cap T_{3} \neq \emptyset$ and thus we may suppose that $f_{i_{l+1}} \in T_{3}$. As $a\left(e_{i_{l}}\right)>0$, there must exist $j \in$ $\left\{i_{l}+1, \ldots, i_{l+1}-1\right\}$ such that $C\left(i_{l}, j\right) \subset T_{3}$ and $A_{j} \cap T_{3} \neq \emptyset$. If not, $T_{3} \backslash\left\{e_{i_{l}}\right\}$ would be in $T(G, S)$, and hence $a\left(e_{i_{l}}\right)=0$, a contradiction. Thus we may suppose $f_{j} \in T_{3}$.

Now, if $C\left(i_{l+1}, j\right) \not \subset T_{3}$, then $\delta(w) \cap T_{3}$ must contain two further edges, and in consequence, the set $\left(T_{3} \backslash\left\{f_{i_{l+1}}, f_{j}\right\}\right) \cup C\left(j, i_{l+1}\right)$ induces a solution of STECSP, yielding

$$
a\left(f_{i_{l+1}}\right)+a\left(f_{j}\right) \leq a\left(C\left(j, i_{l+1}\right)\right)
$$

Also, as by Lemma $3.2(2)$ the set $\left(T^{*} \backslash C\left(j, i_{l+1}\right)\right) \cup\left\{f_{i_{l+1}}, f_{j}\right\}$ belongs to $T(G, S)$, it follows that

$$
a\left(C\left(j, i_{l+1}\right)\right)+a\left(f_{i_{1}}\right) \leq a\left(f_{i_{l+1}}\right)+a\left(f_{j}\right)+a\left(e_{i_{1}}\right) .
$$

By combining (3.6) with (3.5), we get $a\left(f_{i_{1}}\right) \leq a\left(e_{i_{1}}\right)$. But, as $a\left(e_{i_{1}}\right)=0$, it follows that $a\left(f_{i_{1}}\right)=0$, a contradiction.

Thus $C\left(i_{l+1}, j\right) \subset T_{3}$ and consequently, $T_{3}=C\left(i_{l+1}, j\right) \cup\left\{f_{i_{l+1}}, f_{j}\right\}$. Now, we can see that $\left(T_{3} \backslash\left(C\left(i_{l}, j\right) \cup\left\{f_{j}\right\}\right)\right) \cup\left\{f_{i_{l}}\right\}$ induces a solution of the problem. Therefore, $a\left(f_{i_{l}}\right) \geq a\left(C\left(i_{l}, j\right)\right)+a\left(f_{j}\right)$. Since by $(3.4), a\left(f_{i_{j}}\right) \geq a\left(f_{i_{l}}\right)$, it follows that $a\left(C\left(i_{l}, j\right)\right)=0$ and hence $a\left(e_{i_{l}}\right)=0$, a contradiction.

\section{Claim 3.6.}

(1) $a\left(e_{i}\right)=a\left(f_{i}\right)$ for all $i$ different from $i_{1}$ and $i_{1}+1$.

(2) $a\left(e_{i-1}\right)=a\left(f_{i}\right)$ for all $i$ different from $i_{1}+1$ and $i_{1}+2$.

Proof. We will prove (1), the proof of (2) is similar.

By Lemma 3.3 (1), there exists an edge set $T \in t_{a}$ such that $e_{i} \notin T$. As by Claim $3.5 u_{i}, u_{i+1} \in S$, we have $A_{i} \cap T \neq \emptyset$ and $A_{i+1} \cap T \neq \emptyset$. Thus, we may suppose, w.l.o.g., that $f_{i}, f_{i+1} \in T$. We claim that $a\left(e_{i}\right) \geq a\left(f_{i}\right)$. Indeed, if $|\delta(w) \cap T| \geq 3$, then the set $\left(T \backslash\left\{f_{i}\right\}\right) \cup\left\{e_{1}\right\}$ is still a solution of $T(G, S)$ and then $a\left(e_{i}\right) \geq a\left(f_{i}\right)$. If not, $C(i+1, i) \subset T$ and the set $\left(T \backslash\left\{f_{i}\right\}\right) \cup\left\{e_{i}, f_{i_{1}+1}, e_{i_{1}}\right\} \in$ $T(G, S)$, also gives $a\left(e_{i}\right) \geq a\left(f_{i}\right)$. 
Furthermore, by Lemma $3.3(2)$, there exists an edge set $T^{\prime} \in t_{a}$ such that $\left|\delta\left(u_{i+1}\right) \cap T^{\prime}\right| \geq 3$. By Lemmas 3.4 and 3.5, it follows that $C \cup\left\{f_{i+1}\right\} \subset T^{\prime}$. Thus, we may assume that $T^{\prime}=C \cup\left\{f_{i+1}, f_{i_{1}+1}\right\}$. As $\left(T^{\prime} \backslash\left\{e_{i}\right\}\right) \cup\left\{f_{i}\right\} \in T(G, S)$, we have $a\left(f_{i}\right) \geq a\left(e_{i}\right)$ and then $a\left(f_{i}\right)=a\left(e_{i}\right)$.

Claim 3.7. For all edge $f$ of $A_{i}$ such that $i \in\{1, \ldots, n\} \backslash\left\{i_{1}+1\right\}$, we have $a(f)=\beta_{i}$ for some $\beta_{i} \in \mathbb{R}^{+}$.

Proof. The proof is straightforward if $\left|A_{i}\right|=1$. So, suppose $\left|A_{i}\right| \geq 2$. Let us assume, on the contrary, that there is $f^{\prime} \in A_{i}, i \in\{1, \ldots, n\} \backslash\left\{i_{1}+1\right\}$ such that $a\left(f_{i}\right)<a\left(f^{\prime}\right)$. By Lemma $3.3(1)$, there is an edge set $T \in t_{a}$ such that $f^{\prime} \in T$. Hence $f_{i} \in T$, for otherwise $T^{\prime}=\left(T \backslash\left\{f^{\prime}\right\}\right) \cup\left\{f_{i}\right\}$ would be in $T(G, S)$ and $a x^{T^{\prime}}<\alpha$, a contradiction.

We claim that $e_{i-1}, e_{i} \notin T$. Indeed, if $\left|\left\{e_{i-1}, e_{i}\right\} \cap T\right|=1$, then there must exist in $T$ a further edge of $\delta(w)$, different from $f_{i}$ and $f^{\prime}$. In this case, it is easy to see that $T \backslash\left\{f_{i}\right\}$ and $T \backslash\left\{f^{\prime}\right\}$ belong to $t_{a}$ and thus $a\left(f_{i}\right)=a\left(f^{\prime}\right)=0$, a contradiction. Now, assume that $e_{i-1}, e_{i} \in T$. If $C \not \subset T$, then there must exist two further edges of $\delta(w) \backslash\left\{f_{i}, f^{\prime}\right\}$ that belong to $T$. But this implies that $T \backslash\left\{f_{i}, f^{\prime}\right\} \in T(G, S)$, and thus $a\left(f_{i}\right)=a\left(f^{\prime}\right)=0$, a contradiction. If this is not the case, that is $C \subset T$, then $\left(T \backslash\left\{f_{i}\right\}\right) \cup\left\{f_{i_{1}+1}\right\} \in T(G, S)$ and hence $a\left(f_{i}\right)=0$ which yields again a contradiction.

Thus, $e_{i}, e_{i-1} \notin T$. As a consequence, $\left(T \backslash\left\{f^{\prime}\right\}\right) \cup\left\{e_{i}, f_{i_{1}+1}\right\}$ is a set of $T(G, S)$. As $a\left(f_{i_{1}+1}\right)=0$, we obtain that $a\left(e_{i}\right) \geq a\left(f^{\prime}\right)$, and thus $a\left(e_{i}\right)>a\left(f_{i}\right)$, contradicting Claim 3.6 (1).

Combining Claims 3.6 and 3.7, we obtain that

$$
a(e)=\beta, \text { for all } e \in E \backslash\left(\left\{e_{i_{1}}, e_{i_{1}+1}\right\} \cup A_{i_{1}+1}\right) \text { for some } \beta \in \mathbb{R}_{+} .
$$

Claim 3.8. If $A_{i_{1}+1}$ contains at least two edges, then $a(f)=\beta$ for all edge $f$ of $A_{i_{1}+1}$ different from $f_{i_{1}+1}$.

Proof. First of all, note that by Claim $3.2(2)\left(T^{*} \backslash\left\{f_{i_{1}}\right\}\right) \cup\left\{e_{i_{1}}, f\right\}$ belongs to $T(G, S)$ for all $f \in A_{i_{1}+1} \backslash\left\{f_{i_{1}+1}\right\}$. This implies that $a(f) \geq a\left(f_{i_{1}}\right)=\beta$.

On the other hand, for every $f \in A_{i_{1}+1} \backslash\left\{f_{i_{1}+1}\right\}$, by Lemma 3.3 (1) there exists an edge set $T_{f}$ of $t_{a}$ that contains $f$. Clearly, $f_{i_{1}+1} \in T_{f}$. Now, since $\left(T_{f} \backslash\{f\}\right) \cup\left\{e_{i_{1}}, f_{i_{1}}\right\} \in T(G, S)$, we have $a(f) \leq a\left(f_{i_{1}}\right)=\beta$, and hence the claim follows.

Now altogether, we have

$$
\begin{array}{ll}
a(e)=0 & \text { for all } e \in\left\{e_{i_{1}}, e_{i_{1}+1}, f_{i_{1}+1}\right\}, \\
a(e)=\beta & \text { for all } e \in E \backslash\left\{e_{i_{1}}, e_{i_{1}+1}, f_{i_{1}+1}\right\}
\end{array}
$$

This implies that $a x \geq \alpha$ is an inequality of type (2.5) associated to the partition given by the nodes of the graph with $V_{0}=\left\{u_{i_{1}+1}\right\}$, and $F=\left\{e_{i_{1}}, e_{i_{1}+1}, f_{i_{1}+1}\right\}$. 


\subsection{FACETS}

Now we describe necessary conditions for constraints (2.4) and (2.5) to define facets for $\operatorname{STECSP}(G, S)$ on $\boldsymbol{\Gamma}$.

\section{Theorem 3.2.}

(1) The constraints of type (2.4) that may define facets of $\operatorname{STECSP}(G, S)$ are such that

(a) $V_{i}$ contains exactly one terminal for all $i \in\{1, \ldots, p\} \backslash I$,

(b) $F \subset \bigcup_{u_{i} \in S} \delta\left(V_{0}, u_{i}\right)$.

(Recall that $I$ is the set of indices $i$ such that $V_{i}$ does not contain terminal nodes.)

(2) The constraints of type (2.5) that may define facets of $\operatorname{STECSP}(G, S)$ are such that $\left|V_{i} \cap S\right|=1$ for $i=2, \ldots, p$.

Proof. (1)(a) First note that by (2.3), inequality (2.4) can also be written as

$$
x\left(T_{1}\right)+2 x\left(T_{2} \backslash F\right) \geq 2 q-2\left\lfloor\frac{|F|}{2}\right\rfloor .
$$

Now suppose that for some $i \in\{1, \ldots, p\} \backslash I, V_{i}$ contains two terminals. By Condition $C_{2}$ of the generalized Steiner $F$-partition inequality (2.4), these terminals may be supposed consecutive, say $u_{i_{1}}$ and $u_{i_{2}}$. Let $V_{i}^{1}=\left\{u_{j} \in V_{i} \mid j \leq i_{1}\right\}$ and $V_{i}^{2}=V_{i} \backslash V_{i}^{1}$. Let $V_{0}^{\prime}, \ldots, V_{p+1}^{\prime}$ be the partition such that

$$
\begin{aligned}
V_{j}^{\prime} & =V_{j}, \text { for } j=0, \ldots, i-1, \\
V_{i}^{\prime} & =V_{i}^{1}, \\
V_{i+1}^{\prime} & =V_{i}^{2}, \\
V_{j}^{\prime} & =V_{j-1}, \text { for } j=i+2, \ldots, p+1 .
\end{aligned}
$$

Note that this new partition does not satisfy Condition $C_{4}$. Also note that $\delta\left(V_{i}^{1}, V_{i}^{2}\right)=\left\{e_{i_{1}}\right\}$. Hence the generalized Steiner $F$-partition inequality induced by this partition and $F$ can be written as

$$
x\left(T_{1}\right)+2 x\left(\left(T_{2} \cup\left\{e_{i_{1}}\right\}\right) \backslash F\right) \geq 2(q+1)-2\left\lfloor\frac{|F|}{2}\right\rfloor .
$$

It is easy to see that inequality (3.7) can be obtained from (3.8) and the inequality $x\left(e_{i_{1}}\right) \leq 1$. This implies that (3.7) cannot define a facet for $\operatorname{STECSP}(G, S)$.

(1)(b) Suppose that there is an edge $f=w u_{i}$ of $F$ with $u_{i} \notin S$ for some $i \in\{1, \ldots, n\}$. By $(1)\left(\right.$ a) every set $V_{i}, i \in\{1, \ldots, p\} \backslash I$ contains exactly one terminal. Hence $q=s$. Let $V_{j_{k}}$ be the set of the partition that contains terminal $u_{i_{k}}$ for $k=1, \ldots, s$. By Condition $C_{4},\left|F \cap \delta\left(V_{0}, V_{j_{k}}\right)\right|=1$ for $k=1, \ldots, s$, and hence $|F|=s$.

W.l.o.g., we may suppose that $u_{i} \in V_{j_{1}}$. Also we may suppose that $i_{1}<i$, that is node $u_{i_{1}}$ is met before node $u_{i}$ on the cycle $C$. 
Now let us denote (2.4) by $a x \geq \alpha$ and suppose that this inequality defines a non-trivial facet of $\operatorname{STECSP}(G, S)$. Then there must exist an edge set $T \subset E$ not containing $e_{i_{1}}$ and whose incidence vector satisfies (2.4) with equality.

As $|F|$ is odd, we have that $s$, and hence $q$, is odd. Recall that the partition $V_{0}, \ldots, V_{p}$ inducing constraint $(2.4)$, is defined in such a way that between every two consecutive sets containing a terminal, there is at most one set that does not intersect $S$. Let

$$
\Delta_{k}=\delta\left(V_{j_{k}}\right) \cup\left(\bigcup_{\substack{l \in\left\{j_{k}-1, j_{k}+1\right\} \\ V \\ V} S=\emptyset} \delta\left(V_{l}\right)\right)
$$

for $k=2,4, \ldots, q-1$.

Claim. $a\left(\Delta_{k} \cap T\right) \geq 2$ for $k=2,4, \ldots, q-1$.

Proof of the claim. If $T$ contains an edge of $\delta\left(w, V_{j_{k}}\right) \backslash F$, as this edge belongs to $T_{2}$, the claim follows. So suppose that $T \cap\left(\delta\left(w, V_{j_{k}}\right) \backslash F\right)=\emptyset$. As $V_{j_{k}} \cap S \neq \emptyset$ and therefore $T$ contains at least two edges of $\delta\left(V_{j_{k}}\right), T$ must contain at least one edge of $\delta\left(V_{j_{k}}\right) \cap C$. Let $e$ be one of these edges. Suppose, w.l.o.g., that $e \in \delta\left(V_{j_{k}}, V_{j_{k}+1}\right)$.

If $V_{j_{k}+1} \cap S \neq \emptyset$, then $e \in T_{2}$ and hence the claim follows.

If $V_{j_{k}+1} \cap S=\emptyset$, then $e \in T_{1}$ and hence $a(e)=1$. If $e$ is the only edge of $T$ in $\delta\left(V_{j_{k}+1}\right)$, then $T \backslash\{e\}$ still induces a Steiner 2-edge connected subgraph of $G$. But this implies that $a(e)=0$, a contradiction. In consequence, there must exist a further edge, say $e^{\prime}$, of $T$ in $\delta\left(V_{j_{k}+1}\right)$. As $e^{\prime} \in T_{1}$ we have that $a\left(\Delta_{k} \cap T\right) \geq a(e)+a\left(e^{\prime}\right)=2$ which ends the proof of the claim.

Now let $U=\left\{u_{j} \in V_{j_{1}} \mid j \leq j_{1}\right\}$, that is the set of nodes of $V_{j}$ met before $u_{i_{1}+1}$ on the cycle $C$. Note that $f \notin \delta(U)$. As $U \cap S \neq \emptyset$ and $e_{i_{1}} \notin T,\left|T \cap\left(\delta(U) \backslash\left\{e_{i_{1}}\right\}\right)\right| \geq 2$. Let $f_{1}$ and $f_{2}$ be two edges of $T \cap\left(\delta(U) \backslash\left\{e_{i_{1}}\right\}\right)$. Since $f \notin \delta(U)$ and by Condition $C_{4}\left|F \cap \delta\left(V_{j_{1}}\right)\right|=1, f_{1}, f_{2} \in T_{1} \cup\left(T_{2} \backslash F\right)$. Moreover, as $\left|\left(\delta(U) \backslash\left\{e_{i_{1}}\right\}\right) \cap C\right|=1$, at least one of these edges belongs to $T_{2} \backslash F$. In consequence,

$$
a\left(f_{1}\right)+a\left(f_{2}\right) \geq 3 .
$$

In addition, since the sets $\Delta_{k}, k=2,4, \ldots, q-1$ are pairwise disjoint and $\left\{f_{1}, f_{2}\right\}$ does not intersect any of these sets, by the Claim and (3.9), it follows that

$$
a(T) \geq 3+2\left(\frac{q-1}{2}\right)=q+2,
$$

wich is impossible.

(2) Suppose that there is some $i \in\{2, \ldots, p\}$ such that $V_{i}$ contains two terminals. By Condition $C_{2}^{\prime}$ these terminals can be supposed consecutive, say $u_{i_{j}}$ and 
$u_{i_{j+1}}$. Consider the partition $V_{0}^{\prime}, \ldots, V_{p+1}^{\prime}$ given by

$$
\begin{aligned}
V_{k}^{\prime} & =V_{k} \text { for } k=0, \ldots, i-1, \\
V_{i}^{\prime} & =\left\{u_{k} \in V_{i} \mid k \leq i_{j}\right\} \\
V_{i+1}^{\prime} & =\left\{u_{k} \in V_{i} \mid k \geq i_{j}+1\right\} \\
V_{k}^{\prime} & =V_{k-1} \text { for } k=i+2, \ldots, p+1 .
\end{aligned}
$$

Note that $\delta\left(V_{i}^{\prime}, V_{i+1}^{\prime}\right)=\left\{e_{i_{j}}\right\}$. Hence the Steiner $F$-partition inequality induced by this partition and $F$ can be written as

$$
x\left(\Delta \cup\left\{e_{i_{j}}\right\}\right) \geq p .
$$

It is easy to see that inequality (2.5) can be obtained from (3.10) and the trivial inequality $x\left(e_{i_{j}}\right) \leq 1$. This implies that (2.5) cannot define a facet of $\operatorname{STECSP}(G, S)$.

We can easily see that there is a polynomial number of inequalities of type (2.4) and (2.5) that satisfy the conditions of Theorem 3.2, and thus define facets of $\operatorname{STECSP}(G, S)$, when $G$ is in $\boldsymbol{\Gamma}$. Thus we have the following.

Corollary 3.1. The separation problem for inequalities (2.4) and (2.5) that define facets of $\operatorname{STECSP}(G, S)$, when $G$ is a graph of $\boldsymbol{\Gamma}$, can be solved in polynomial time.

\section{Composition OF GRAPHS}

In this section, we shall discuss some applications of the previous results to a class of graphs containing Halin graphs.

A 3-edge cutset is a cut with 3 edges. If $G$ is a graph having a 3-edge cutset $\delta(W)$, then $G$ decomposes into $G_{1}$ and $G_{2}$ where $G_{1}\left(G_{2}\right)$ is obtained by shrinking $W(V \backslash W)$ to a single node. In the sequel, we will denote by $\boldsymbol{\Omega}$ the set of graphs that decomposes by 3 -edge cutsets into graphs of $\boldsymbol{\Gamma}$ and series-parallel graphs. We can note that a Halin graph decomposes into wheels by 3 -edge cutsets. Thus $\boldsymbol{\Omega}$ contains the class of Halin graphs.

In [4], Barahona and Mahjoub show that if $G$ decomposes into $G_{1}$ and $G_{2}$ by a 3 -edge cutset $\delta(W)$, then a system that defines $\operatorname{STECSP}(G, V)$ is obtained from the union of the systems that define $\operatorname{STECSP}\left(G_{1}, V\right)$ and $\operatorname{STECSP}\left(G_{2}, V\right)$ and by identifying the variables associated with the edges in $\delta(W)$. As a consequence, they obtain that STECSP $(G, S)$ is completely given by the trivial, Steiner cut and Steiner $F$-partition inequalities when $S=V$ and $G$ is a Halin graph.

Such a composition cannot, unfortunately, be applied for the $\operatorname{STECSP}(G, S)$ when $S \neq V$. However, in this case we have the following analogous result. The proof is similar to that given in [4] when $S=V$.

Theorem 4.1. Let $G=(V, E)$ be a graph that has a 3-edge cutset $\delta(W)=$ $\left\{u_{i_{1}} u_{j_{1}}, u_{i_{2}} u_{j_{2}}, u_{i_{3}} u_{j_{3}}\right\}$ and $S \subset V$ a set of terminals. Suppose that $u_{i_{1}}, u_{i_{2}}$, $u_{i_{3}}, u_{j_{1}}, u_{j_{2}}, u_{j_{3}} \subseteq S$. Let $G_{1}=\left(V_{1}, E_{1}\right)$ and $G_{2}=\left(V_{2}, E_{2}\right)$ be obtained from $G$ 


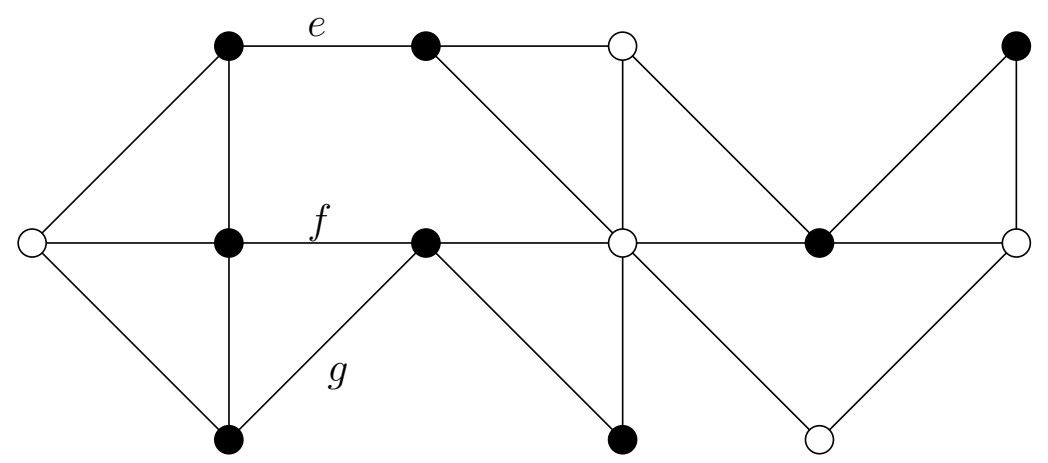

FiguRe 5.

by shrinking $W$ and $V \backslash W$, respectively. Then a system of linear inequalities sufficient to define $\operatorname{STECSP}(G, S)$ is obtained from the union of the systems that define $\operatorname{STECSP}\left(G_{1}, S_{1}\right)$ and $\operatorname{STECSP}\left(G_{2}, S_{2}\right)$, and by identifying the variables associated with the edges in $\delta(W)$. Here $S_{1}=S \backslash W \cup\left\{w_{1}\right\}$ and $S_{2}=S \backslash(V \backslash W) \cup\left\{w_{2}\right\}$ where $w_{1}\left(w_{2}\right)$ is the node that arises from the contraction of $W(V \backslash W)$.

In [3], Baïou and Mahjoub show that the polytope $\operatorname{STECSP}(G, S)$ is given by the trivial and Steiner cut inequalities when $G$ is series-parallel. As a consequence of this result and Theorems 3.1 and 4.1, we have the following.

Corollary 4.1. Let $G=(V, E)$ be a graph of $\boldsymbol{\Omega}$. Suppose that the endnodes of the edges of the 3-edge cutsets involved in the decomposition of $G$ are all terminals. Then $\operatorname{STECSP}(G, S)$ is given by the trivial, Steiner cut and generalized Steiner $F$-partition inequalities.

Corollary 4.1 generalizes the result of Barahona and Mahjoub [4] when $S=V$. To illustrate Corollary 4.1, consider the graph $G=(V, E)$ shown in Figure 5 where the terminals are filled nodes and Steiner nodes are the others.

Graph $G$ can be decomposed, by the 3-edge cutset formed by the edges $e, f$ and $g$, in the wheel $W$ and the serie-parallel graph $S P$ of Figure 6 .

It follows, by Corollary 4.1, that $\operatorname{STECSP}(G, S)$ is given by the trivial and Steiner cut inequalities describing the polytope $\operatorname{STECSP}(S P, S)$ and the trivial, Steiner cut and generalized Steiner $F$-partition inequalities defining the polytope of the wheel $W$.

As mentioned above, the composition used in Theorem 4.1 cannot be applied when the endnodes of the edges of the 3-edge cutset are not all in $S$. As it will turn out, the generalized $F$-partition inequalities, the basic trivial and the Steiner cut inequalities do not suffice to completely describe the polytope $\operatorname{STECSP}(G, S)$ in this case. In fact, consider for instance the graph $G=(V, E)$ shown in Figure 7 where $S=\left\{u_{1}, u_{3}, u_{4}, u_{6}\right\}$.

Let $x$ be the solution given by $x\left(e_{1}\right)=x\left(e_{3}\right)=x\left(e_{8}\right)=x\left(e_{9}\right)=1$ and $x\left(e_{2}\right)=$ $x\left(e_{4}\right)=x\left(e_{5}\right)=x\left(e_{6}\right)=x\left(e_{7}\right)=1 / 2$. It is not hard to see that this solution 

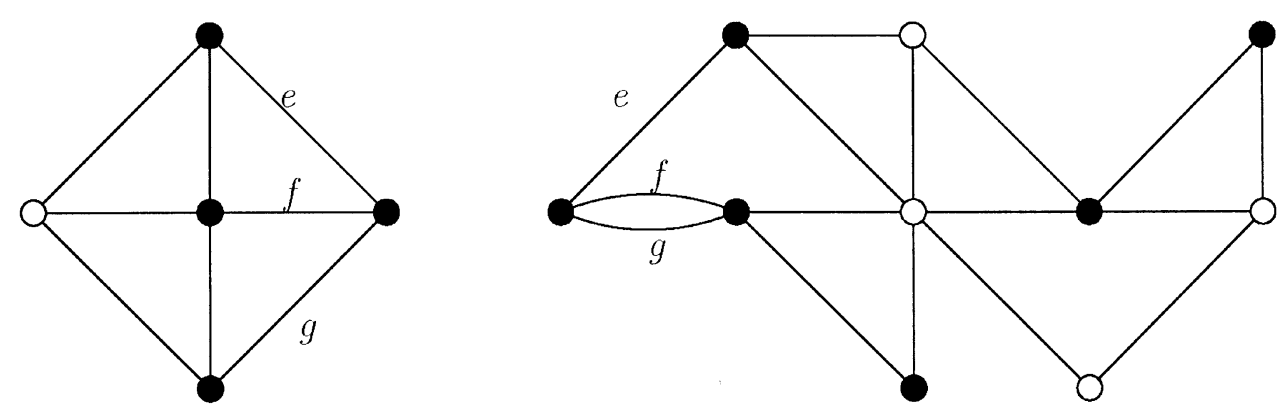

FIGURE 6 .

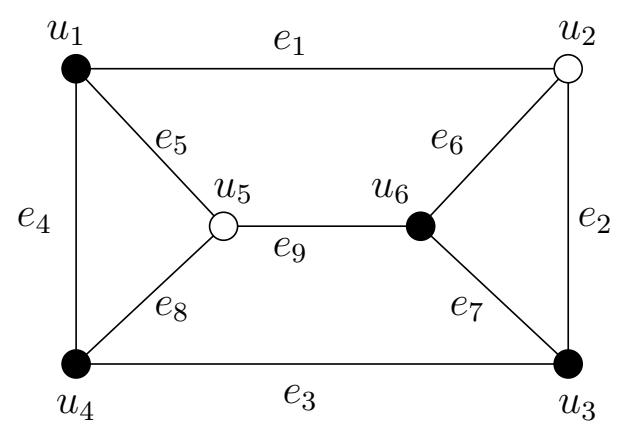

FIGURE 7 .

satisfies all the trivial, Steiner cut and generalized Steiner F-partition inequalities. Moreover, $x$ is an extreme point of the polytope given by these inequalities.

\section{Algorithmic aspects}

The decomposition given in the previous section has a practical interest for the problem of finding a minimum cost Steiner 2-edge connected subgraph of a graph of $\boldsymbol{\Omega}$. Indeed, let $G$ be a graph of $\boldsymbol{\Omega}$. If this graph is neither a graph of $\boldsymbol{\Gamma}$ nor a serie-parallel graph, it then decomposes by a 3-edge cutset $\{e, f, g\}$ into two graphs $G_{1}$ and $G_{2}$ of $\Omega$. If the endnodes of the edges $e, f$ and $g$ are all terminals, then, we can obtain an optimal solution of the STECSP on $G$ using the following procedure. This uses ideas similar to those used by Barahona and Mahjoub in [4].

Let us denote by $\lambda\left(F_{1}, F_{2}, G_{2}\right)$ the value of an optimal solution of STECSP on the graph $G_{2}$ containing the edges of $F_{1}$ and not the ones of $F_{2}$ where the weights on the edges of $G_{2}$ are taken to be the same as for $G$. The problem is solved in $G_{1}$ where the edge weights are the same as for $G$ except for $e, f$ and $g$ for which 
the weights are taken to be the solution of the following linear system

$$
\begin{aligned}
& w_{e}^{\prime}+w_{f}^{\prime}=\lambda\left(\{e, f\},\{g\}, G_{2}\right)-\kappa \\
& w_{f}^{\prime}+w_{g}^{\prime}=\lambda\left(\{f, g\},\{e\}, G_{2}\right)-\kappa \\
& w_{e}^{\prime}+w_{g}^{\prime}=\lambda\left(\{e, g\},\{f\}, G_{2}\right)-\kappa \\
& w_{e}^{\prime}+w_{f}^{\prime}+w_{g}^{\prime}=\lambda\left(\{e, f, g\}, \emptyset, G_{2}\right)-\kappa
\end{aligned}
$$

where $w_{e}^{\prime}, w_{f}^{\prime}$ and $w_{g}^{\prime}$ are the new weights and $\kappa$ is a variable guaranteeing the solvability of the system. Such a solution contains necessarily either 2 or 3 edges of $\{e, f, g\}$. An optimal solution of STECSP on $G$ can be obtained by recomposing the optimal solution obtained on $G_{1}$ and an optimal solution of $G_{2}$ that contains exactly the same edges of $\{e, f, g\}$ that are contained in the solution on $G_{1}$. The value of the opimal solution thus obtained is $\lambda\left(G_{1}\right)+\kappa$ where $\lambda\left(G_{1}\right)$ is the value of the optimal solution of STECSP obtained on $G_{1}$.

It follows that solving the STECSP on a graph of $\boldsymbol{\Omega}$ can be reduced in solving the problem on graphs of $\boldsymbol{\Gamma}$ or on series-parallel graphs. If $G$ is a serie-parallel graph, then $\operatorname{STECSP}(G, S)$ is completly described by the trivial and Steiner cut inequalities. As these inequalities can be separated in polynomial time, one can solve the problem on $G$ using a cutting plane algorithm.

Suppose now that $G$ is a graph of $\boldsymbol{\Gamma}$. By Theorem 3.1 the polytope $\operatorname{STECSP}(G, S)$ is given by the trivial and Steiner cut inequalities together with inequalities (2.4) and (2.5). By Corollary 3.1, it follows that the problem STECSP can be solved in polynomial time on $G$ using a cutting plane algorithm.

Given a graph of $\boldsymbol{\Omega}$, one can compute in polynomial time (using 3-edge cutsets) the pieces of $G$. In consequence, problem STECSP can be solved in polynomial time by cutting planes on the class of $\boldsymbol{\Omega}$ when the endnodes of the edges of the 3-edge cutsets are all terminals. To the best of our knowledge, this is the first polynomial time algorithm for STECSP on a class that generalizes the class of Halin graphs.

\section{Concluding Remarks}

In this paper we have discussed the Steiner 2-edge connected subgraph polytope. We have introduced a new class of valid inequalities for this polytope that generalizes the so-called Steiner $F$-partition inequalities. We have shown that these inequalities together with the trivial and the Steiner cut inequalities completely describe the Steiner 2-edge connected subgraph polytope on a class of graphs that generalizes the wheels. We have also discussed some necessary conditions for these inequalities to be facet defining. As a consequence, we have obtained that the facet defining inequalities of this type can be separated in polynomial time on that class of graphs. Furthermore, we have discussed the polytope $\operatorname{STECSP}(G, S)$ in the graphs that decompose by 3 -edge cutsets. We have shown that the generalized Steiner $F$-partition inequalities together with the trivial and the Steiner cut inequalities suffice to describe the polytope $\operatorname{STECSP}(G, S)$ on a class of graphs 
containing Halin graphs, when the nodes of all the 3-edge cutsets of $G$ are terminals. This generalizes the result of Barahona and Mahjoub [4] when all the nodes of the graph are terminals. This is, to the best of our knowledge, the first complete description of $\operatorname{STECSP}(G, S)$ given for a nontrivial class of graphs that includes inequalities other than the basic trivial and Steiner cut inequalities. In contrast to the case where $S=V$, the additional inequalities may be non-rank inequalities. Moreover, we have addressed the algorithmic aspect and shown that the STECSP can be solved in polynomial time on that class of graphs using cutting plane algorithm, when the endnodes of the edges in the 3-edge cutsets are all terminals.

The fact that the generalized Steiner $F$-partition inequalities suffice, with the trivial and the Steiner cut inequalities, to characterize the polytope $\operatorname{STECSP}(G, S)$ in a nontrivial class of graphs, shows, as is the case for the $F$-partition inequalities $[1,24]$, that these inequalities may be of great interest in solving the STECSP within the framework of a cutting plane algorithm. For this, it would be interesting to characterize the generalized Steiner $F$-partition inequalities that define facets. It would also be interesting to investigate extensions of the results of the paper to the Steiner $k$-edge connected subgraph problem.

Acknowledgements. We would like to thank the anonymous referee for his/her constructive comments.

\section{REFERENCES}

[1] M. Baïou, Le problème du sous-graphe Steiner 2-arête connexe: approche polyédrale. Ph.D. thesis, Université de Rennes 1 (1996).

[2] M. Baïou, F. Barahona and A.R. Mahjoub, Separation of partition inequalities. Math. Oper. Res. 25 (2000) 243-254.

[3] M. Baïou and A.R. Mahjoub, Steiner 2-edge connected subgraph polytopes on series-parallel graphs. SIAM J. Discrete Math. 10 (1997) 505-514.

[4] F. Barahona and A.R. Mahjoub, On two-connected subgraph polytopes. Discrete Math. 147 (1995) 19-34.

[5] D. Bienstock, E.F. Brickell and C.L. Monma, On the structure of minimum-weight $k$ connected spanning networks. SIAM J. Discrete Math. 3 (1990) 320-329.

[6] S.C. Boyd and T. Hao, An integer polytope related to the design of survivable communication networks. SIAM J. Discrete Math. 6 (1993) 612-630.

[7] S. Chopra, The k-edge connected spanning subgraph polyhedron. SIAM J. Discrete Math. 7 (1994) 245-259.

[8] N. Christofides and C.A. Whitlock, Network synthesis with connectivity constraints: a survey, in Operational Research 81, Hamburg edited by J.P. Brans (1981) 705-723.

[9] G. Cornuéjols, J. Fonlupt and D. Naddef, The traveling salesman problem on a graph and some related integer polyhedra. Math. program. 33 (1985) 1-27.

[10] R. Coullard, A. Rais, R.L. Rardin and D.K. Wagner, Linear-time algorithm for the 2connected Steiner subgraph problem on special classes of graphs. Networks 23 (1993) 195206.

[11] R. Coullard, A. Rais, R.L. Rardin and D.K. Wagner, The dominant of the 2-connected steiner subgraph polytope for $W_{4}$-free graphs. Discrete Appl. Math. 66 (1996) 33-43. 
[12] M. Didi Biha and A.R. Mahjoub, $k$-edge connected polyhedra on series-parallel graphs. Oper. Res. Lett. 19 (1996) 71-78.

[13] M. Didi Biha and A.R. Mahjoub, The $k$-edge connected subgraph problem I: Polytopes and critical extreme points. Linear Algebra Appl. 381 (2004) 117-139.

[14] R.E. Erikson, C.L. Monma, and Jr. A.F. Veinott, Send and split method for a minimumconcave-cost network flows. Math. Oper. Res. 12 (1987) 634-664.

[15] J. Fonlupt and A.R. Mahjoub, Critical extreme points of the 2-edge connected subgraph polytope, in Integer Programming and Combinatorial Optimization: 7 th International IPCO Conference, edited by G. Cornuéjols, R.E. Burkard and G.J. Woeginger, Lect. Notes Comput. Sci. Graz, Austria, Springer-Verlag 1610 (1999) 166-183.

[16] J. Fonlupt and A.R. Mahjoub, Critical extreme points of the 2-edge connected spanning subgraph polytope. Math. Program. 105 (2006) 289-310.

[17] J. Fonlupt and D. Naddef, The traveling salesman problem in graphs with some excluded minors. Math. Program. 53 (1992) 147-172.

[18] M. Grötschel and C. Monma, Integer polyhedra arising from certain network design problems with connectivity constraints. SIAM J. Discrete Math. 3 (1990) 502-523.

[19] M. Grötschel, C. Monma and M. Stoer, Polyhedral approaches to network survivability, in Reliability of computer and Communication Networks, edited by F. Hwang F. Roberts and C. Monma, Series Discrete Math. Comput. Sci. 5 (1991) 121-141 AMS/ACM.

[20] M. Grötschel, C. Monma and M. Stoer, Computational results with a cutting plane algorithm for designing communication networks with low-connectivity constraints. Oper. Res. 40 (1992) 309-330.

[21] M. Grötschel, C. Monma and M. Stoer, Facets for polyhedra arising in the design of communication networks with low-connectivity constraints. SIAM J. Optim. 2 (1992) 474-504.

[22] M. Grötschel, C.L. Monma and M. Stoer, Design of Survivable Networks, in Network Models, Handbooks in Operations Research and Management Science, Elsevier, North-Holland, Amsterdam, Vol. 7, chapter 10 (1995) 617-672.

[23] R. Halin, Studies on minimality n-connected graphs. In Combinatorial Math. Appl., edited by J.A. Welsh, Academic Press, New York (1971) 129-136.

[24] H. Kerivin, Réseaux fiables et polyèdres. Ph.D. thesis, Université de Clermont Ferrand II, France (2000).

[25] H. Kerivin and A.R. Mahjoub, Design of survivable networks: A survey. Networks 46 (2005) $1-21$.

[26] H. Kerivin, A.R. Mahjoub and C. Nocq, (1,2)-survivable networks: Facets and branch-andcut, in The Sharpest Cut, edited by M. Grötschel, MPS-SIAM Series in Optimization (2004) $121-152$.

[27] H. Kerivin and A. Ridha Mahjoub, On survivable network polyhedra. Discrete Math. 290 (2005) 183-210.

[28] A.R. Mahjoub, Two-edge connected spanning subgraphs and polyhedra. Math. Program. 64 (1994) 199-208.

[29] A.R. Mahjoub and P. Pesneau, On the Steiner 2-edge connected subgraph polytope. Technical Report RR-06:02, LIMOS, Université Blaise Pascal, Clermont-Ferrand, France (2006).

[30] C. Monma, B. Munson and W. Pulleyblank, Minimum-weight two-connected spanning networks. Math. Program. 46 (1990) 153-171.

[31] W.R. Pulleyblank, Polyhedral Combinatorics, OR \& MS, in Optimization, volume 1, North Holland, Amsterdam (1989) 371-446.

[32] K. Steiglitz, P. Weinen and D.J. Kleitmann, The design of minimum cost survivable networks. IEEE Trans. Circuit Theory 16 (1969) 455-460.

[33] S. Voss, Steiner-probleme in graphen. Math. Systems in Economics (1990).

[34] P. Winter, Generalized Steiner problem in Halin graphs, in Proceedings of the 12th International Symposium on Math. Program. MIT (1985).

[35] P. Winter, Generalized Steiner problem in series-parallel networks. J. Algor. 7 (1986) 549566. 